\title{
Acta Botanica Mexicana
}

\section{Floristic checklist from the hills La Cantera and Delgado, Jantetelco, Morelos, Mexico}

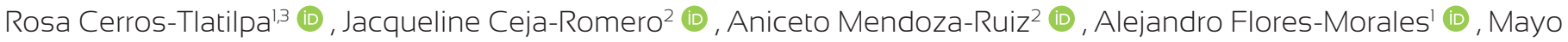 \\ Jaramillo-Sánchez'
}

\section{Resumen:}

Antecedentes y Objetivos: No obstante, el intenso trabajo florístico y taxonómico que se ha realizado en el estado de Morelos, aún hay áreas poco exploradas. En este estudio se presenta el inventario florístico de las plantas vasculares que crecen en los cerros La Cantera y Delgado, localizados dentro de la zona arqueológica de Chalcatzingo, municipio Jantetelco, Morelos, México.

Métodos: Para la realización del trabajo, durante los años 2014 a 2017 se hicieron salidas de campo para recolectar ejemplares fértiles, los cuales fueron secados y posteriormente identificados con claves especializadas. La determinación taxonómica se verificó con material de herbario, y en algunos casos fue confirmada por especialistas.

Resultados clave: En total se registraron 85 familias, 285 géneros, 434 especies con 27 categorías infraespecíficas de plantas vasculares. Las eudicotiledóneas fueron el grupo más diverso con 66 familias, 212 géneros y 307 especies, seguidas por las monocotiledóneas con diez familias, 57 géneros y 100 especies, las pteridofitas con ocho familias, 15 géneros y 25 especies y las magnólidas con una familia, un género y dos especies. También se reportan nueve nuevos registros a nivel específico para el estado.

Conclusiones: La zona de estudio alberga $42.7 \%$ de las familias, $26.6 \%$ de los géneros y $12.4 \%$ de las especies registradas hasta el momento para Morelos, lo que confirma la importancia no solo cultural sino también biológica del sitio, y pone de manifiesto la necesidad de continuar con la exploración botánica en esta parte de Morelos.

Palabras clave: angiospermas, bosque tropical caducifolio, nuevos registros, riqueza.

\begin{abstract}
: municipality of Jantetelco, Morelos, Mexico. specialists. also reported at a specific level for the state. Atlixco Núm. 186, Colonia Vicentina, Iztapalapa, 09340 Ciudad de México, México.

${ }^{3}$ Autor para la correspondencia: tlatilpa@uaem.mx
\end{abstract}

Background and Aims: Nevertheless, the intense floristic and taxonomic work in the Morelos state, there are some areas little explored. In this study a floristic inventory of vascular plants is presented from the hills Delgado and La Cantera, located at the archeological zone of Chalcatzingo, in the

Methods: In order to carry out the work, during the years 2014 to 2017, fieldwork was conducted to collect fertile specimens, which were dried and later identified with specialized keys. The taxonomic identification was verified with herbarium specimens, and in some cases was confirmed by

Key results: A total of 85 families, 285 genera, 434 species with 27 infraspecific categories of vascular plants were recorded. Eudicotyledons were the most diverse group with 66 families, 212 genera and 307 species, followed by monocots with ten families, 57 genera and 100 species, the pteridophytes with eigth families, 15 genera and 25 species and the magnoliides with one family, one genera and two species. In addition, 9 new records are

Conclusions: The study area records $42.7 \%$ of the families, $26.6 \%$ of the genera and $12.4 \%$ of the species registered so far from Morelos, which confirms the cultural and biological importance of this site and highlights the need to continue with the botanical exploration in this part of Morelos. Key words: angiosperms, new records, pteridophytes, richness, tropical deciduous forest.
Recibido: 29 de mayo de 2019

Revisado: 25 de junio de 2019.

Aceptado por Rosario Redonda-Martínez: 13 de septiembre de 2019.

Publicado Primero en línea: 30 de octubre de 2019.

Publicado: Acta Botanica Mexicana 127 (2020).
Citar como:

Cerros-Tlatilpa, R., J. Ceja-Romero, A. Mendoza-Ruiz, A. Flores-Morales y M. Jaramillo-Sánchez. 2019(2020). Inventario florístico de los cerros La Cantera y Delgado, Jantetelco, Morelos, México. Acta Botanica Mexicana 127: e1565. DOI: 10.21829/abm127.2020.1565
Este es un artículo de acceso abierto (c) (1) $\$$ by
bajo la licencia Creative Commons 4.0
Atribución-No Comercial (CC BY-NC 4.0 Internacional).
e-ISSN: 2448-7589 


\section{Introducción}

México es uno de los países con mayor diversidad vegetal en el mundo, debido a la abrupta topografía que presenta y su amplio mosaico de climas (Villaseñor, 2003), lo que le confiere también un alto grado de endemismo (Rzedowski, 1991). En 2016, Villaseñor estimó que en el territorio habi$\tan 23,314$ especies de plantas vasculares, distribuidas en 2854 géneros (49.8\% de ellos endémicos) y 297 familias. Las angiospermas fueron el grupo más diverso con 22,126 especies, le siguieron las pteridofitas y plantas afines con 1039 y por último las gimnospermas con 149.

El estado de Morelos se ubica entre las regiones biogeográficas Neártica y Neotropical, además forma parte de dos zonas consideradas centros de endemismo, la Faja Volcánica Transmexicana y la Cuenca del Balsas (Suárez-Mota et al., 2013; Guerrero et al., 2015). No obstante que es una de las entidades más pequeñas de México con 4941 km² de superficie, alberga el $14.9 \%$ de las especies de fanerógamas (Villaseñor, 2016), convirtiéndose así en un importante reservorio de la diversidad vegetal.

Entre los trabajos florísticos para plantas vasculares que hay en Morelos, a nivel estatal están los realizados por Vázquez (1974) y Bonilla-Barbosa y Villaseñor (2003), en este último se registran 198 familias, 1016 géneros y 3345 especies. En particular para pteridofitas y grupos afines, se cuenta con el de Riba et al. (1996), quienes reportaron 158 especies, 50 géneros y 21 familias en la entidad. También existen trabajos taxonómicos para las familias Orchidaceae (Espejo-Serna et al., 2002), Onagraceae (Miguel-Vázquez y Cerros-Tlatilpa, 2013), Apocynaceae (González-Rocha y Cerros-Tlatilpa, 2015) y Poaceae (Sánchez-Ken y Cerros Tlatilpa, 2016), así como una serie de inventarios regionales (Soria, 1985; Cerros-Tlatilpa y Espejo-Serna, 1998; Hernández-Cárdenas et al., 2014) y estudios para la Reserva de la Biosfera de Sierra de Huautla (Dorado et al., 2005), el Corredor Biológico Chichinautzin (Flores-Castorena y MartínezAlvarado, 2011) y el Parque Nacional El Tepozteco (Block y Meave, 2015). A pesar del trabajo florístico y taxonómico que se ha realizado en el estado, todavía hay zonas poco exploradas (Guerrero et al., 2015), por lo que es imperativo a corto y mediano plazo documentar la diversidad biológica que se presenta en esos sitios (Villaseñor, 2003), sobre todo porque la entidad se está modificando y deteriorando de forma acelerada, con la consiguiente pérdida de cubierta vegetal (Cerros-Tlatilpa y Espejo-Serna, 1998).

Trejo y Dirzo (2000) estimaron que el bosque tropical caducifolio (sensu Rzedowski, 2006), tipo de vegetación dominante en Morelos, se redujo en poco más de $60 \%$ de su cobertura original entre 1973 y 1989. Esta asociación vegetal, se caracteriza por presentar dos estaciones bien marcadas, la lluviosa y la seca, esta última con una duración de cinco a ocho meses, durante la cual las plantas pierden sus hojas, debido a que sus suelos son someros y pedregosos, no son los mejores para la agricultura; sin embargo, en zonas de fuerte presión demográfica han sido sometidos al cultivo de manera extensiva (Rzedowski, 2006), situación que se observa en Jantetelco, que de acuerdo con Dorado et al. (2015), se encuentra entre los municipios que están en la categoría de deforestados.

Jantetelco forma parte de las áreas de importancia agrícola de Morelos, con $80.08 \%$ de su superficie destinada para tal fin (INEGI, 2009). En la parte central del municipio se localiza la zona arqueológica de Chalcatzingo, resguardada por los cerros La Cantera y Delgado, elevaciones en las que debido a su abrupta topografía se conservan restos de bosque tropical caducifolio, tipo de vegetación que ocupa apenas el 9.52\% del territorio municipal (INEGI, 2009), cuya sobrevivencia se encuentra amenazada por el incremento demográfico del poblado, que ha comenzado a traspasar los límites inferiores del sitio (Grove, 2008; INEGI, 2010), y por el deterioro derivado de la actividad turística que se lleva a cabo en el área. Ante este panorama, y dada la importancia cultural y biológica de este lugar, el presente trabajo tuvo como objetivo realizar el inventario de las plantas vasculares que crecen de manera silvestre en los cerros La Cantera y Delgado, contribuyendo con ello al conocimiento florístico de la entidad y de México.

\section{Materiales y Métodos}

\section{Área de estudio}

Los cerros La Cantera y Delgado (Fig. 1) se localizan en la Zona Arqueológica de Chalcatzingo, en el municipio Jantetelco, Morelos, México; abarca alrededor de 40 ha y se ubica entre las coordenadas $18^{\circ} 40^{\prime} 19.3^{\prime \prime} \mathrm{N}$ y $98^{\circ} 45^{\prime} 55.9^{\prime \prime O} \mathrm{O}$. El municipio colinda al norte con el estado de Puebla y los municipios Ayala y Temoac; al este con el estado de Puebla 


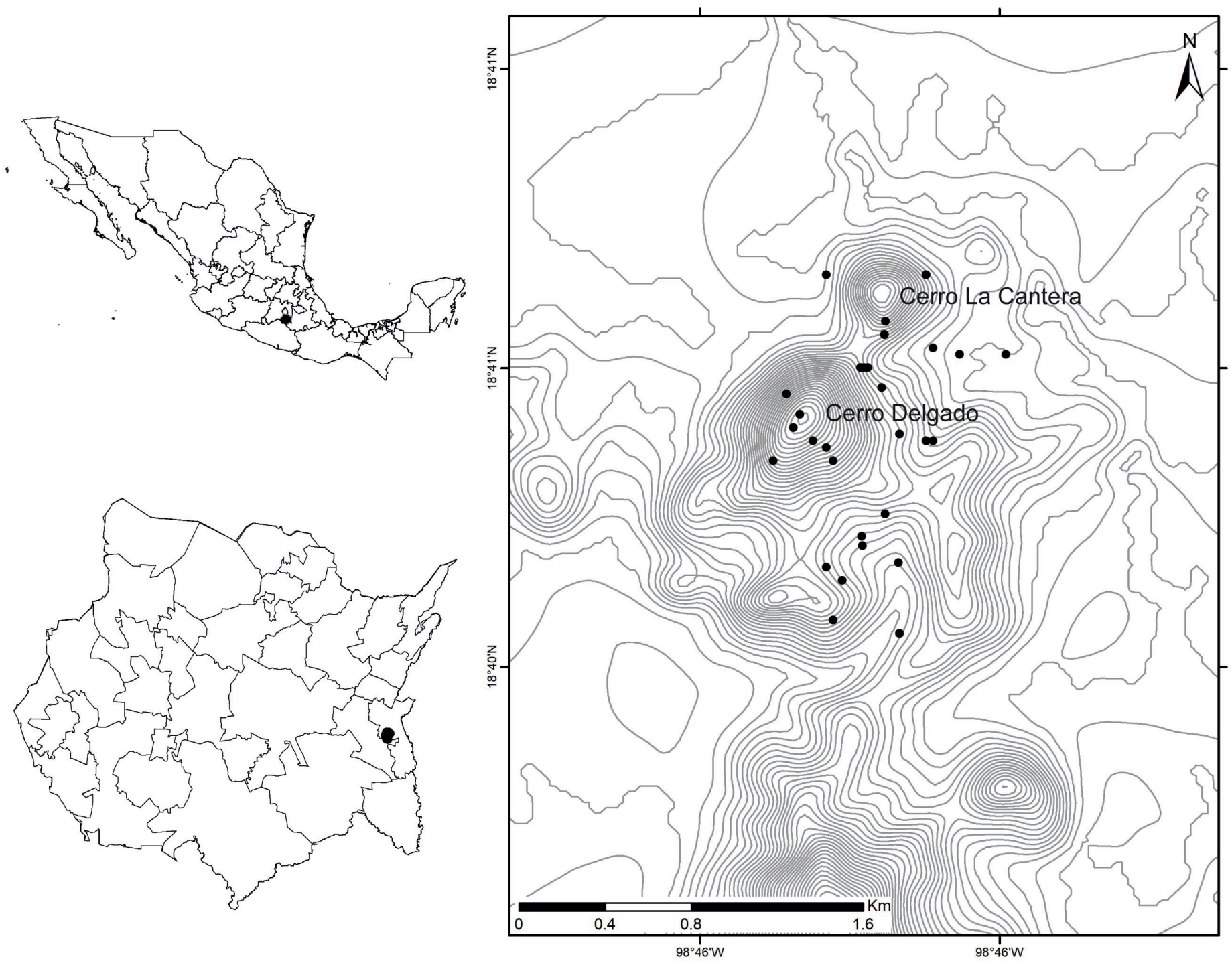

Figura 1: Localización de los cerros La Cantera y Delgado, Jantetelco, Morelos, México (círculos negros señalan los sitios de colecta).

y el municipio Axochiapan; al sur con los municipios Axochiapan y Jonacatepec; y al oeste con los municipios Ayala y Jonacatepec (INEGI, 2009). Los cerros que circundan el sitio son afloramientos constituidos por restos de cuellos volcánicos compuestos por rocas ígneas intrusivas hipoabisales (Illescas y Buitrago, 2015), con suelos tipo Leptosol y Regosol (INEGI, 2016), tienen una precipitación media anual de $927.5 \mathrm{~mm}$, clima cálido sub-húmedo, temperatura anual máxima de $29.6{ }^{\circ} \mathrm{C}$, mínima de $12.2^{\circ} \mathrm{C}$ y media de $20.9{ }^{\circ} \mathrm{C}$ (INEGI, 2009). El tipo de vegetación presente corresponde al bosque tropical caducifolio (Figs. 2A-F).

\section{Trabajo de campo y procesamiento de las especies}

Se realizaron de manera periódica salidas de campo de 2014 a 2017, que abarcaron las épocas secas y de lluvias, con el fin de recolectar material fértil de angiospermas y pteridofitas. Los recorridos se hicieron de manera aleatoria desde la parte más baja hasta la cima de ambos cerros, a lo largo de veredas y zonas escarpadas, recolectando muestras de las especies que se encontraban con estructuras reproductivas. Los ejemplares se herborizaron de acuerdo a Germán (1986) y se identificaron con claves especializadas (Calderón de Rzedowski, 1992; Ramírez y Téllez, 1992; Tucker, 1994; Pérez-Calix, 2008; González-Rocha y Cerros- 

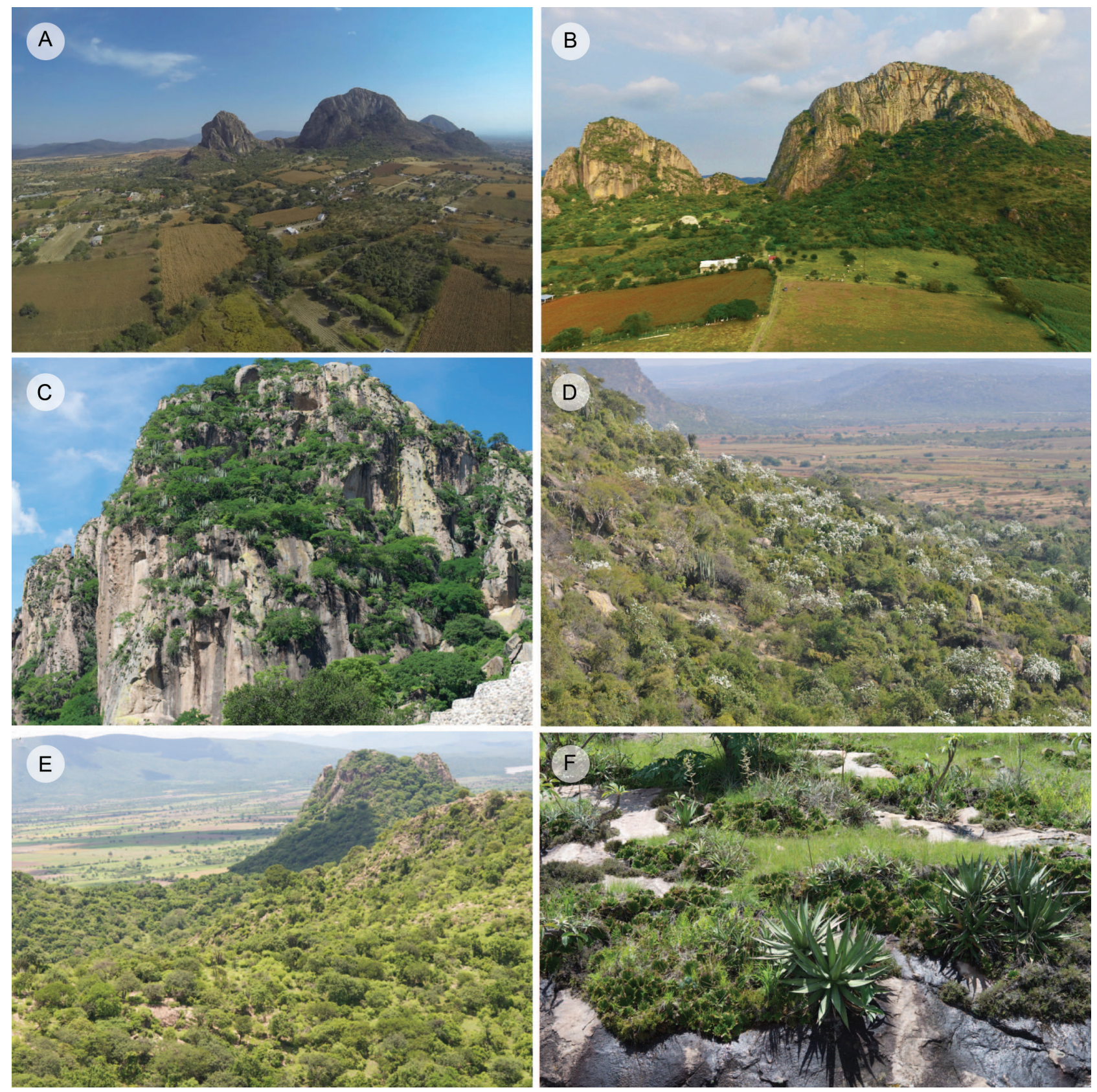

Figura 2: Apariencia general de los cerros La Cantera y Delgado, Jantetelco, Morelos, México. A-B. vista aérea de los cerros Delgado y La Cantera; C. vista este del cerro Delgado; D. vista oeste del cerro Delgado; E. vista oeste del cerro La Cantera; F. ladera del cerro La Cantera. Fotografías: María Luisa Alquicira y Rosa Cerros-Tlatilpa.

Tlatilpa, 2015), incluyendo claves digitales como FAMEX (ABACo A.C., 2015), Neotropikey (Milliken et al., 2009+) y Flora of North America (FNA, 1993+). La determinación fue cotejada con material depositado en los herbarios de la Universidad Autónoma del Estado de Morelos (HUMO), Universidad Nacional Autónoma de México (MEXU) y de la Universidad Autónoma Metropolitana, Iztapalapa (UAMIZ), así como con fotografías digitales de tipos disponibles en sitios electrónicos como Journal Storage Global Plants (JSTOR, 2019), The New York Botanical Garden (NYBG, 2018), Muséum National D'Histoire Naturelle (MNHN, 2018), Smithsonian National Museum of Natural History (SNMNH, 2018), Royal Botanical Gardens (RBGK, 2019), Jardín Botánico de Missouri (TROPICOS, 2019). En algunos casos se solicitó el apoyo de especialistas para corroborar la identidad de las especies, cabe señalar que los nombres de 
las familias de pteridofitas y angiospermas se actualizaron de acuerdo con la clasificación de APG III, APG IV y PPG I (APG, 2009, 2016; PPGI, 2016). Los ejemplares de respaldo están depositados en los herbarios HUMO, BUAP (Benemérita Universidad Autónoma de Puebla), MEXU y UAMIZ.

\section{Resultados}

En el área de estudio se identificaron 85 familias, 285 géneros, 434 especies con 27 categorías infraespecíficas de plantas vasculares (Apéndice). Las eudicotiledóneas son el grupo más diverso con 66 familias, 212 géneros y 307 especies, seguidas por las monocotiledóneas con diez familias, 57 géneros y 100 especies, las pteridofitas con ocho familias, 15 géneros y 25 especies y las magnólidas con una familia, un género y dos especies (Cuadro 1, Figs. 3-6).

Las familias con mayor número de géneros y especies fueron Poaceae, Fabaceae, Asteraceae, Euphorbia-

Cuadro 1: Número de géneros y especies por familia, de las plantas vasculares presentes en los cerros La Cantera y Delgado, Jantetelco, Morelos, México. El asterisco $\left({ }^{*}\right)$ indica las familias con mayor riqueza de taxa.

\begin{tabular}{|c|c|c|}
\hline Familias & Géneros & Especies \\
\hline PTERIDOFITAS Y AFINES & 15 & 25 \\
\hline Anemiaceae & 1 & 2 \\
\hline Aspleniaceae & 1 & 1 \\
\hline Dennstaedtiaceae & 1 & 1 \\
\hline Dryopteridaceae & 1 & 1 \\
\hline Ophioglossaceae & 1 & 1 \\
\hline Pteridaceae * & 8 & 14 \\
\hline Selaginellaceae & 1 & 4 \\
\hline Thelypteridaceae & 1 & 1 \\
\hline MONOCOTILEDÓNEAS & 57 & 100 \\
\hline Amaryllidaceae & 3 & 3 \\
\hline Araceae & 1 & 2 \\
\hline Asparagaceae & 5 & 6 \\
\hline Bromeliaceae * & 4 & 10 \\
\hline Commelinaceae & 5 & 9 \\
\hline Cyperaceae & 4 & 8 \\
\hline Iridaceae & 1 & 2 \\
\hline Marantaceae & 1 & 1 \\
\hline Orchidaceae & 6 & 6 \\
\hline Poaceae * & 27 & 53 \\
\hline MAGNÓLIDAS & 1 & 2 \\
\hline Piperaceae & 1 & 2 \\
\hline EUDICOTILEDÓNEAS & 212 & 307 \\
\hline Acanthaceae * & 9 & 13 \\
\hline
\end{tabular}

Cuadro 1: Continuación

\begin{tabular}{|c|c|c|}
\hline Familias & Géneros & Especies \\
\hline Amaranthaceae & 5 & 7 \\
\hline Anacardiaceae & 2 & 2 \\
\hline Annonaceae & 1 & 1 \\
\hline Apiaceae & 1 & 1 \\
\hline Apocynaceae * & 11 & 14 \\
\hline Araliaceae & 1 & 1 \\
\hline Asteraceae * & 36 & 46 \\
\hline Begoniaceae & 1 & 1 \\
\hline Bignoniaceae & 3 & 3 \\
\hline Brassicaceae & 1 & 1 \\
\hline Burseraceae & 1 & 4 \\
\hline Cactaceae * & 7 & 11 \\
\hline Campanulaceae & 2 & 2 \\
\hline Caryophyllaceae & 1 & 2 \\
\hline Cleomaceae & 1 & 1 \\
\hline Convolvulaceae & 2 & 8 \\
\hline Cordiaceae & 1 & 3 \\
\hline Crassulaceae & 2 & 2 \\
\hline Cucurbitaceae & 2 & 2 \\
\hline Ebenaceae & 1 & 1 \\
\hline Euphorbiaceae * & 7 & 15 \\
\hline Fabaceae * & 31 & 49 \\
\hline Gentianaceae & 1 & 1 \\
\hline Heliotropiaceae & 3 & 3 \\
\hline Hernandiaceae & 1 & 1 \\
\hline Lamiaceae & 2 & 4 \\
\hline Lennoaceae & 1 & 1 \\
\hline Linderniaceae & 1 & 1 \\
\hline Loasaceae & 2 & 3 \\
\hline Loranthaceae & 3 & 4 \\
\hline Lythraceae & 2 & 3 \\
\hline Malpighiaceae & 5 & 5 \\
\hline Malvaceae * & 11 & 15 \\
\hline Meliaceae & 1 & 2 \\
\hline Menispermaceae & 1 & 1 \\
\hline Molluginaceae & 1 & 1 \\
\hline Moraceae & 1 & 3 \\
\hline Myrtaceae & 1 & 1 \\
\hline Namaceae & 1 & 2 \\
\hline Nyctaginaceae & 3 & 4 \\
\hline Onagraceae & 3 & 4 \\
\hline Opiliaceae & 1 & 1 \\
\hline Oxalidaceae & 1 & 1 \\
\hline Papaveraceae & 1 & 1 \\
\hline Passifloraceae & 1 & 2 \\
\hline Petiveriaceae & 1 & 1 \\
\hline Picramniaceae & 1 & 1 \\
\hline Plantaginaceae & 3 & 3 \\
\hline Plumbaginaceae & 1 & 2 \\
\hline Polemoniaceae & 1 & 2 \\
\hline Polygalaceae & 2 & 3 \\
\hline
\end{tabular}


Cuadro 1: Continuación

\begin{tabular}{lcc}
\hline Familias & Géneros & Especies \\
\hline Polygonaceae & 2 & 2 \\
Portulacaceae & 1 & 3 \\
Ranunculaceae & 1 & 2 \\
Rubiaceae & 7 & 9 \\
Rutaceae & 1 & 1 \\
Salicaceae & 2 & 3 \\
Santalaceae & 1 & 2 \\
Sapindaceae & 2 & 3 \\
Scrophulariaceae & 1 & 1 \\
Solanaceae & 2 & 6 \\
Talinaceae & 1 & 1 \\
Urticaceae & 1 & 1 \\
Verbenaceae & 3 & 5 \\
Vitaceae & 1 & 2 \\
\hline Total & 285 & 434 \\
\hline
\end{tabular}

ceae, Malvaceae, Apocynaceae, Pteridaceae, Acanthaceae, Cactaceae y Bromeliaceae; en conjunto representan $52.6 \%$ de los géneros y $55.3 \%$ de las especies registradas en el presente trabajo; en contraste 29 familias están representadas por un solo taxón (Cuadro 1). Los géneros con más riqueza específica fueron Bouteloua Lag., Tillandsia L., Ipomoea L., Euphorbia L., Senna Mill., Bursera Jacq. ex L., Cyperus L., Muhlenbergia Schreb. y Selaginella P. Beauv. (Cuadro 2).

En lo que se refiere a la forma de crecimiento, las hierbas fueron las más abundantes con 260 especies (59.9\%), seguidas de los arbustos con 63 (14.5\%), los árboles con $46(10.6 \%)$ y las trepadoras con 41 (9.4\%), en menor cantidad se presentaron epífitas, parásitas, acuáticas, candelabriformes, cilíndricas y globosas, que en conjunto constituyen apenas 5.5\% (Cuadro 3). Por familia, el mayor número de especies herbáceas se presentaron en Poaceae (53) y Asteraceae (35); arbustivas en Fabaceae (20), Asteraceae (11) y Cactaceae (cinco); arbóreas en Fabaceae (nueve), Burseraceae (cuatro) y Euphorbiaceae (cuatro), y trepadoras en Apocynaceae (diez), Fabaceae (nueve) y Convolvulaceae (cinco). La mayoría de las epífitas pertenecen a Bromeliaceae, particularmente al género Tillandsia, y las parásitas a Loranthaceae y Santalaceae.

De las 434 especies 101 son endémicas de México y dos se restringen a Morelos, Tillandsia chalcatzingensis González-Rocha, Cerros, López-Ferr. \& Espejo y Viridantha rzedowskiana Hern.-Cárdenas, Espejo y López-Ferr. (Apéndice). Tres taxa se encuentran en la NOM-059-SEMAR-
NAT-2010 (SEMARNAT, 2010): Mammillaria magnifica F.G. Buchenau (Cactaceae), sujeta a protección especial; Sapium macrocarpum Müll. Arg. (Euphorbiaceae) y Coryphantha elephantidens (Lem.) Lem. (Cactaceae), en la categoría de amenazadas.

El 3.22\% del total de especies registradas en este trabajo corresponde a 14 especies exóticas (Villaseñor y Espinosa-García, 2004; Espinosa-García et al., 2009, Sánchez-Ken et al., 2012). La familia con mayor número de representantes no nativos es Poaceae con diez, mientras que las cuatro restantes tienen sólo uno (Cuadro 4). Además, cabe señalar que 34 especies de Poaceae se comportan como malezas (Sánchez-Ken et al., 2012).

Se presentan nueve registros nuevos para el estado: Cleome guianensis Aubl. (Cleomaceae), Varronia guanacastensis (Standl.) J.S. Mill. (Cordiaceae), Cyperus amabilis Vahl. (Cyperaceae), Allosidastrum pyramidatum (Desp. ex Cav.) Krapov., Fryxell \& D.M. Bates, Wissadula excelsior (Cav.) C. Presl (Malvaceae), Odontocarya mexicana Barneby (Menispermaceae), Nama jamaicensis L. (Namaceae), Sporobolus tenuissimus (Schrank.) Kuntze (Poaceae) y Notholaena lemmonii D.C. Eaton var. australis R.M. Tryon (Pteridaceae) (Cuadro 5).

\section{Discusión}

El bosque tropical caducifolio en el estado de Morelos representa cerca de $73 \%$ de la superficie arbolada de la entidad (Boyas et al., 2001); sin embargo, estimaciones indican que alrededor de $60 \%$ se ha perdido o transformado (Trejo y Dirzo, 2000). El municipio de Jantetelco ocupa $1.85 \%$ de la superficie del estado, de la cual el bosque tropical caducifolio abarca sólo $9.52 \%$ de su territorio (INEGI, 2009) y se considera fuertemente perturbado (Boyas et al., 2001; Dorado et al., 2005). A pesar de ello, los resultados obtenidos en este trabajo muestran que los cerros La Cantera y Delgado, localizados en la zona arqueológica de Chalcatzingo, albergan $42.7 \%$ de las familias, $26.6 \%$ de los géneros y $12.4 \%$ de las especies reportadas por Villaseñor (2016) para la entidad, lo que refleja la importancia del sitio, sobre todo si tenemos en cuenta que, como lo han señalado algunos autores (Trejo y Dirzo, 2002; Rzedowski y Calderón de Rzedowski, 2013), la composición florística de esta asociación vegetal en nuestro país con frecuencia discrepa de una localidad a otra. 

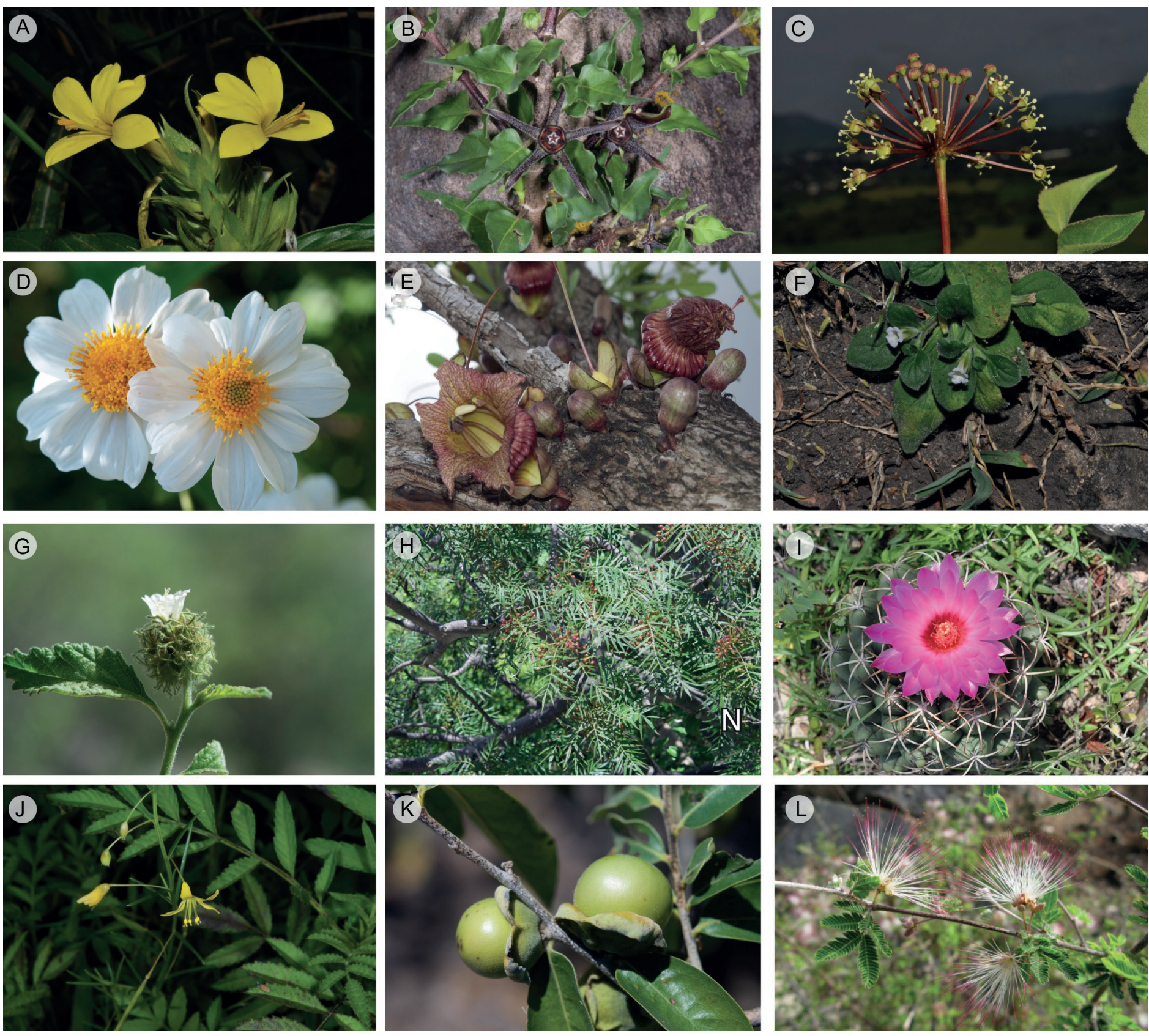

Figura 3. Eudicotiledóneas representativas de los cerros La Cantera y Delgado, Jantetelco, Morelos, México. A. Barleria oenotheroides Dum. Cours.; B. Matelea trachyantha (Greenm.) W.D. Stevens; C. Aralia humilis Cav.; D. Montanoa grandiflora (DC.) Sch. Bip. ex Hemsl.; E. Crescentia alata Kunth; F. Nama jamaicensis L.; G. Varronia guanacastensis (Standl.) J.S. Mill.; H. Bursera bicolor (Willd. ex Schltdl.) Engl.; I. Coryphanta elephantidens (Lem.) Lem.; J. Cleome guianensis Aubl.; K. Diospyros salicifolia Humb. \& Bonpl. ex Willd. L. Calliandra eriophylla Benth. Fotografías: Rosa Cerros-Tlatilpa.

Una gran parte del trabajo florístico realizado en Morelos se ha concentrado en la Reserva de la Biósfera Sierra de Huautla y el Corredor Biológico Chichinautzin (Dorado et al., 2005; Pulido-Esparza et al., 2009; Flores-Castorena y Martínez-Alvarado, 2011; Block y Meave, 2015), áreas cuya extensión comprende varios municipios y tipos de vegetación, por lo que al comparar la riqueza específica aquí registrada con la de éstas y otras zonas del estado para las que se ha generado información (Cuadro 6), es necesario tener en cuenta que la diferencia entre ellas puede estar relacionada con los factores antes mencionados, y el esfuerzo de colecta. Por ejemplo, de los 368 taxa que habitan en los cerros El Sombrerito y Las Mariposas en el municipio Tlayacapan (Cerros-Tlatilpa y Espejo-Serna, 1998), sólo 251 

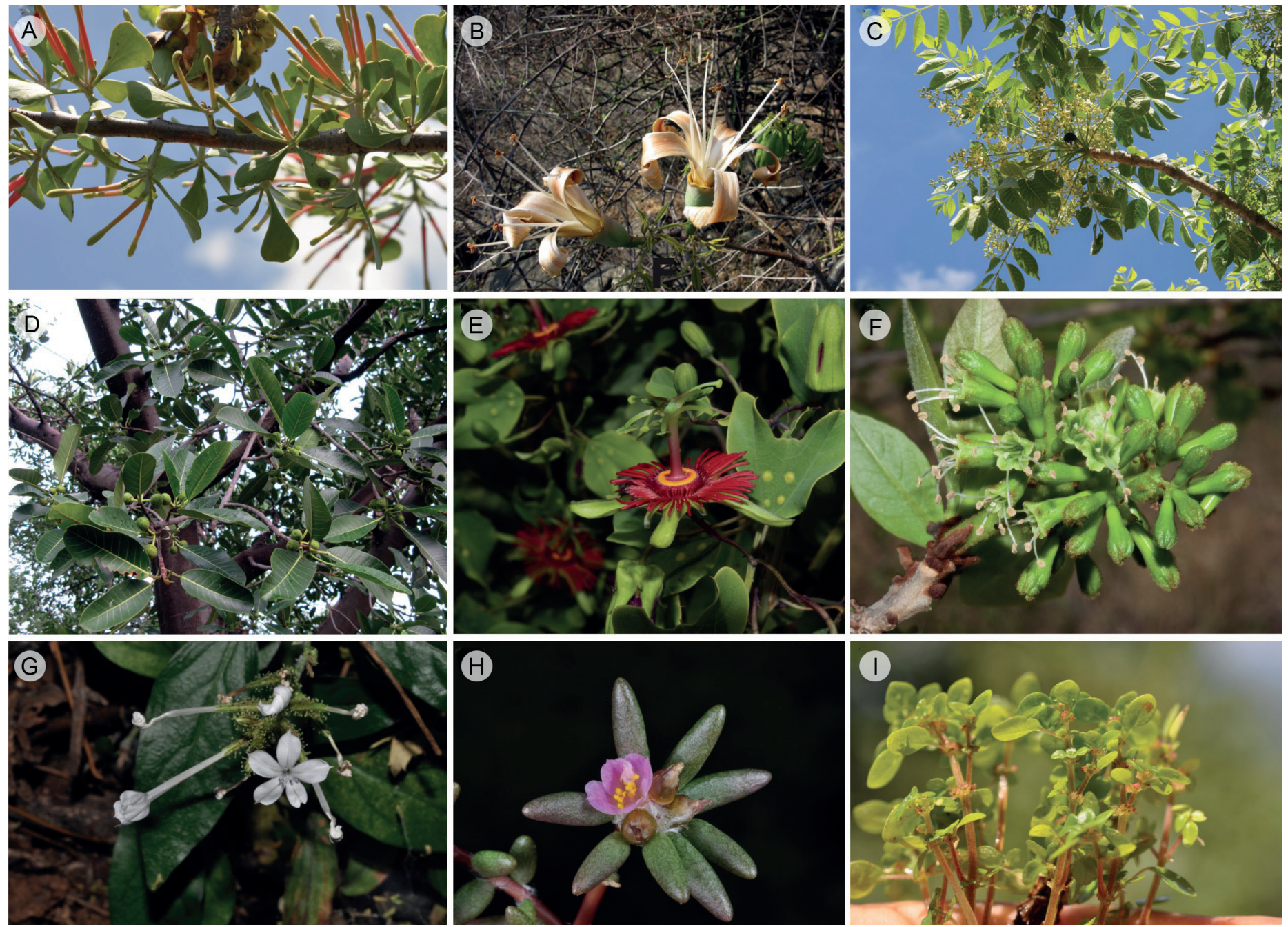

Figura 4: Eudicotiledóneas representativas de los cerros La Cantera y Delgado, Jantetelco, Morelos, México. A. Psittacanthus palmeri (S. Watson) Barlow \& Wiens; B. Ceiba aesculifolia (Kunth) Britten \& Baker f. subsp. parvifolia (Rose) P.E. Gibbs \& Semir; C. Trichilia hirta L.; D. Ficus crocata (Miq.) Mart. ex Miq.; E. Passiflora mexicana Juss.; F. Guapira petenensis (Lundell) Lundell; G. Plumbago zeylanica L.; H. Portulaca pilosa L.; I. Pilea microphylla (L.) Liebm. Fotografías: Rosa Cerros-Tlatilpa.

crecen en bosque tropical caducifolio, el resto se encuentra en bosque de pino-encino y vegetación secundaria. De manera similar, en la barranca Tepecapa, municipio Tlayacapan (Hernández-Cárdenas et al., 2014), de las 434 especies registradas, únicamente 98 de ellas crecen en este tipo de vegetación. La riqueza por grupo taxonómico se puede apreciar en el caso de las monocotiledóneas, ya que PulidoEsparza et al. (2009), reportaron 100 taxa para el corredor biológico del Chichinautzin, mientras que para el área de estudio se obtuvo un listado de 101.

Ocho de las diez familias con mayor número de especies, Poaceae, Fabaceae, Asteraceae, Malvaceae, Euphorbiaceae, Apocynaceae, Acanthaceae y Cactaceae, fueron señaladas como características del bosque tropical caducifolio en México (Rzedowski y Calderón de Rzedowski, 2013), además se ha relacionado la presencia de esta última con el aspecto más seco de este tipo de vegetación. Las otras dos familias que sobresalen por su riqueza específica son Pteridaceae y Bromeliaceae, respecto a la primera, Rzedowski (2006) indicó que, si bien las pteridofitas son poco frecuentes en el bosque tropical caducifolio, pueden presentar cierta abundancia en taludes rocosos, situación que ocurre en el área de estudio, donde crecen principalmente en zonas sombreadas de cañadas y taludes, aunque Astrolepis sinuata (Lag. ex Sw.) D.M. Benham \& Windham y Myriopteris aurea (Poir.) Grusz \& Windham lo hacen tam- 

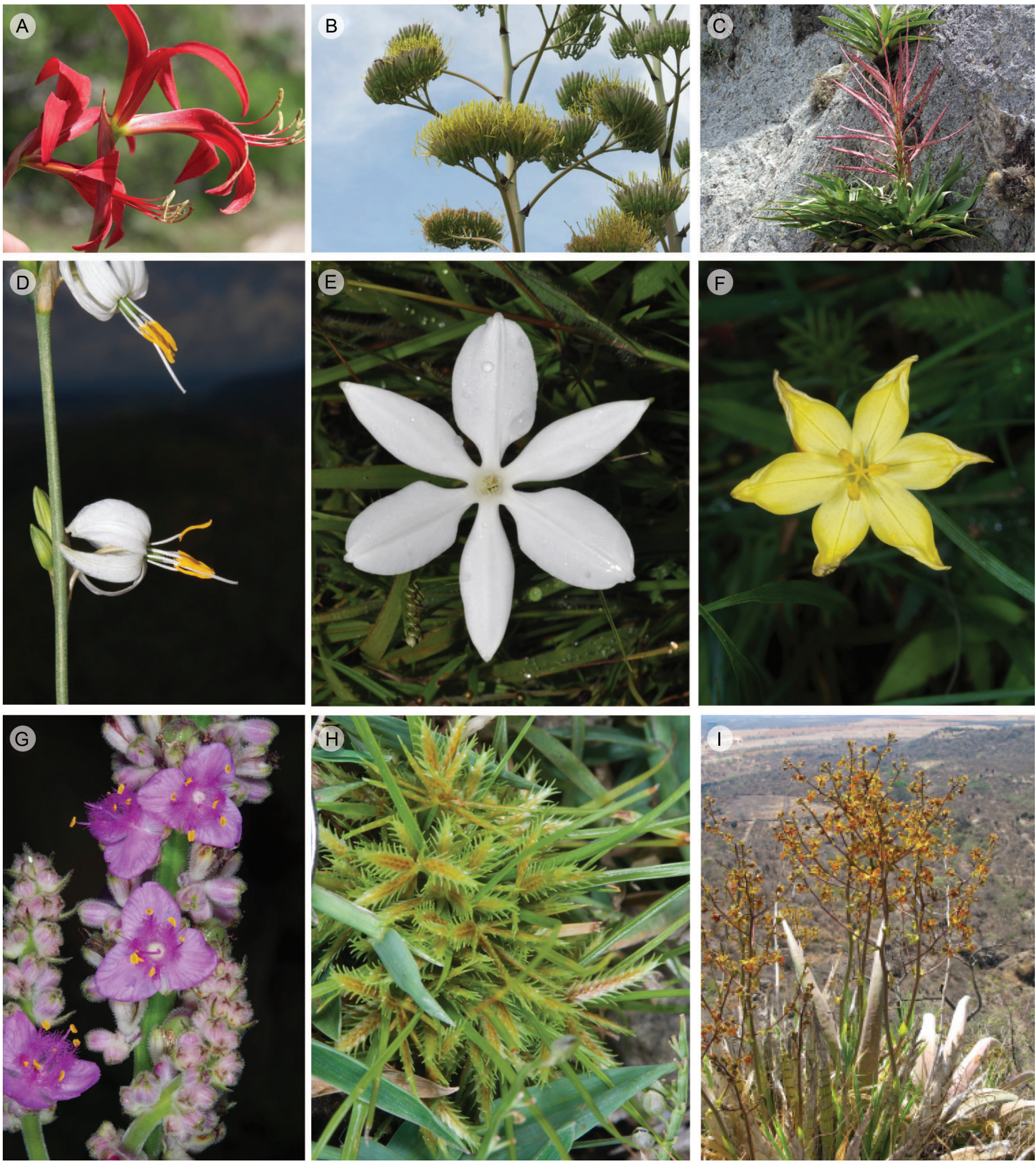

Figura 5: Monocotiledóneas representativas de los cerros La Cantera y Delgado, Jantetelco, Morelos, México. A. Sprekelia formosissima (L.) Herb.; B. Agave vivipara Haw.; C. Tillandsia chalcatzingensis González-Rocha, Cerros, López-Ferr. \& Espejo; D. Echeandia albiflora (Cham. \& Schltdl.) M. Martens \& Galeotti; E. Milla biflora Cav.; F. Sisyrinchium tenuifolium Humb. \& Bonpl. ex Willd.; G. Thyrsanthemum macrophyllum (Greenm.) Rohweder; H. Cyperus squarrosus L.; I. Cyrtopodium macrobulbon (Lex.) G.A. Romero \& Carnevali. Fotografías: Rosa Cerros-Tlatilpa. 

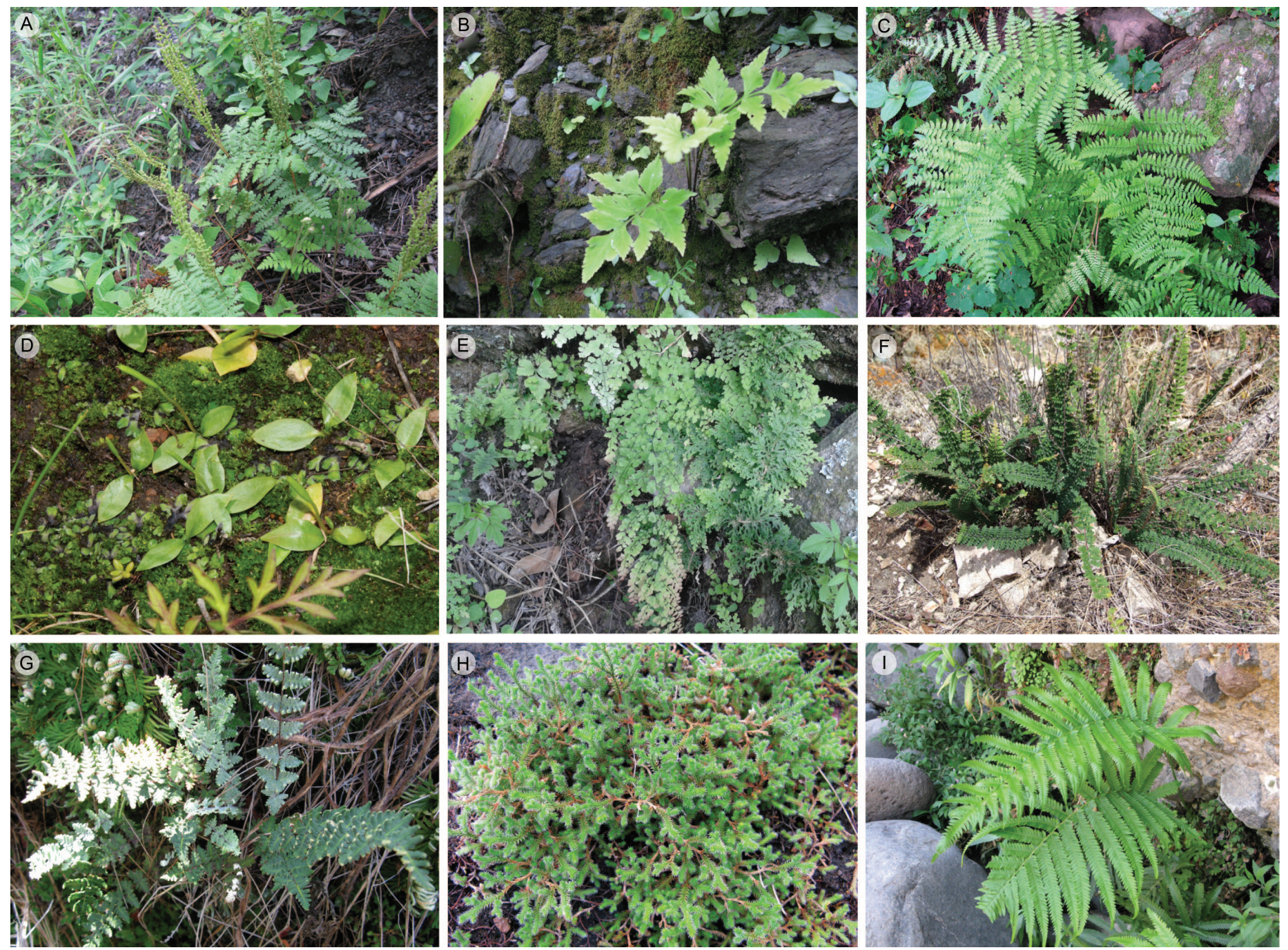

Figura 6: Pteridofitas de los cerros La Cantera y Delgado, Jantetelco, Morelos, México. A. Anemia tomentosa (Savigny) Sw. var. mexicana (C. Presl) Mickel; B. Asplenium pumilum Sw.; C. Dryopteris karwinkyana (Mett.) Kunze; D. Ophioglossum nudicaule L. f.; E. Adiantum concinnum Humb. \& Bonpl. ex Willd.; F. Astrolepis sinuata (Lag. ex Sw.) D.M. Benham \& Windham; G. Notholaena lemmonii D.C. Eaton var. australis R.M. Tryon; H. Selaginella sartorii Hieron.; I. Thelypteris kunthii (Desv.) C.V. Morton. Fotografias: Aniceto Mendoza-Ruiz.

Cuadro 2: Géneros con mayor número de especies en en los cerros La Cantera y Delgado, Jantetelco, Morelos, México.

\begin{tabular}{lc}
\hline Géneros & Especies \\
\hline Bouteloua Lag & 8 \\
Tillandsia L. & 7 \\
Ipomoea L. & 7 \\
Euphorbia L. & 6 \\
Senna Mill. & 5 \\
Bursera Jacq. ex L. & 4 \\
Cyperus L. & 4 \\
Muhlenbergia Schreb. & 4 \\
Selaginella P. Beauv. & 4 \\
\hline
\end{tabular}

Cuadro 3: Número de especies y porcentaje por forma de crecimiento en los cerros La Cantera y Delgado, Jantetelco, Morelos, México. A: árbol, Ac: acuática, Can: candelabriforme, Ar: arbusto, C: cilíndrica, E: epífita, G: globosa, $\mathrm{H}$ : hierba, P: parásita, T: trepadora.

\begin{tabular}{lcc}
\hline $\begin{array}{l}\text { Forma de } \\
\text { crecimiento }\end{array}$ & Número de especies & Porcentaje \% \\
\hline A & 46 & 10.6 \\
Ac & 3 & 0.7 \\
Can & 3 & 0.7 \\
Ar & 63 & 14.5 \\
C & 2 & 0.5 \\
E & 8 & 1.8 \\
G & 1 & 0.2 \\
H & 260 & 59.9 \\
P & 7 & 1.6 \\
T & 41 & 9.4 \\
\hline Total & 434 & 100 \\
\hline
\end{tabular}


bién en lugares expuestos. En cuanto a Bromeliaceae, el mayor aporte específico lo hace Tillandsia, taxon citado por Rzedowski y Calderón de Rzedowski (2013), como uno de los géneros con más especies que se distribuyen de forma preferencial en el bosque tropical caducifolio.

Los cerros La Cantera y Delgado presentan un porcentaje relevante de endemismo con $7.8 \%$ del total de especies con esta categoría, esto con base en los datos que registró Villaseñor (2016) para el estado, sobre todo si consideramos que ambos cerros representan remanentes aislados del bosque tropical caducifolio. Es probable que además contribuya el hecho de que la vegetación se desarrolla en áreas de difícil acceso como taludes y laderas rocosas, características que hacen que el suelo sea inadecuado para las actividades agrícolas y ganaderas, lo que ha permitido su conservación y el descubrimiento de nuevos taxa, tales como Tillandsia chalcatzingensis (González-Rocha et al., 2015) y Viridantha rzedowskiana (Hernández-Cárdenas et al., 2018), que fueron descritas y se conocen únicamente de los alrededores de la zona arqueológica de Chalcatzingo.

En lo concerniente a las especies que se encuentran en alguna categoría de riesgo de acuerdo con la NOM059-SEMARNAT-2010 (SEMARNAT, 2010), Mammillaria magnifica y Coryphantha elephantidens, son endémicas de México. La primera se distribuye en Puebla y Morelos, está sujeta a protección especial, mientras que la segunda, se registra en catorce estados del país (Villaseñor, 2016) y se considera amenazada. Por otra parte, aunque Sapium macrocarpum se distribuye de México a Centro América, también se le considera en la categoría amenazada.

La mayoría de las especies introducidas que se presentan en los cerros La Cantera y Delgado proceden del Viejo Mundo (Cuadro 3) y son herbáceas. Poaceae aporta el mayor número de taxones dentro de esta categoría con diez (13.7\%) de los 73 que se tienen registrados para el estado (Sánchez-Ken y Cerros-Tlatilpa, 2016), lo cual no es de extrañar si consideramos que a nivel nacional es la familia con más especies no nativas en México, seguida de Fabaceae y Asteraceae (Villaseñor y Espinosa-García, 2004), las cuales también están representadas en la zona con una especie cada una. Se considera que la introducción de pastos forrajeros, que posteriormente escaparon del cultivo es una de las posibles causas para la prevalencia de Poaceae en lo que a número de especies exóticas se refiere (Villaseñor y Espinosa-García, 2004). Por otra parte, las especies que se registran por primera vez para Morelos habitan

Cuadro 4: Especies exóticas en los cerros La Cantera y Delgado, Jantetelco, Morelos, México.

\begin{tabular}{llc}
\hline Familia & Especie & Distribución nativa \\
\hline Cyperaceae & Cyperus esculentus L. & Eurasia \\
Poaceae & Bothriochloa pertusa (L.) A. Camus & E y S de Asia \\
& Cenchrus polystachios (L.) Morrone & Estados Unidos, Centro y \\
Sudamérica, África & N de África, S de Europa \\
& Cynodon dactylon (L.) Pers. & Estados Unidos, Centro y \\
& Digitaria bicornis (Lam.) Roem. \& Schult. & Sudamérica, Asia \\
& Echinochloa crus-galli (L.) P. Beauv. & Europa, India \\
& Eleusine indica (L.) Gaertn. & África, Asia, Europa \\
& Eragrostis pilosa (L.) P. Beauv. & Eurasia \\
Eragrostis viscosa (Retz.) Trin. & Trópicos del viejo mundo \\
Asteraceae & Hyperthelia dissoluta (Nees ex Steud.) Clayton & África, Madagascar \\
Molluginaceae & Melinis repens (Willd.) Zizka & África \\
\hline
\end{tabular}


Cuadro 5: Distribución conocida en México de los nuevos registros en los cerros La Cantera y Delgado, Jantetelco, Morelos, México. Baja California (BC), Baja California Sur (BCS), Campeche (CAM), Chiapas (CHIS), Chihuahua (CHIH), Coahuila (COAH), Colima (COL), Durango (DGO), Guerrero (GRO), Guanajuato (GTO), Hidalgo (HGO), Jalisco (JAL), Estado de México (MEX), Michoacán (MICH), Nayarit (NAY), Nuevo León (NL), Oaxaca (OAX), Puebla (PUE), Querétaro (QRO), Quintana Roo (QROO), San Luis Potosí (SLP), Sinaloa (SIN), Sonora (SON), Tabasco (TAB), Tamaulipas (TAM), Veracruz (VER), Yucatán (YUC), Zacatecas (ZAC).

\begin{tabular}{lll}
\hline Familia & Especie & Distribución en México \\
\hline Pteridaceae & Notholaena lemmonii D.C. Eaton var. australis R.M. Tryon & GRO, MEX, MICH, OAX, PUE \\
Cyperaceae & Cyperus amabilis Vahl & BCS, CHIS, CHIH, COL, DGO, GRO, JAL, MEX, MICH, \\
Poaceae & NAY, OAX, SLP, SIN, SON, TAB, VER, ZAC \\
Cleomaceae & Cleome guianensis Aubl. & CHIS, MICH \\
Cordiaceae & Varronia guanacastensis (Standl.) J.S. Mill. & BCS, CHIS, GRO, NAY, OAX, SON, VER \\
Malvaceae & Allosidastrum pyramidatum (Desp. ex Cav.) Krapov., Fryxell \& & CHIS, DGO, GRO, NAY, OAX \\
& D.M. Bates & PUE, QRO, QROL, GRO, HGO, JAL, MICH, NAY, OAX, \\
& Wissadula excelsior (Cav.) C. Presl & CAM, CHIS, GRO, OAX, PUE, SLP, TAB, VER \\
Menispermaceae & Odontocarya mexicana Barneby & CHIS, GRO, OAX, PUE, VER \\
& & BCS, CAM, CHIS, CHIH, COAH, COL, GTO, HGO, \\
Namaceae & Nama jamaicensis L. & JAL, MICH, NL, OAX, PUE, QRO, QROO, SLP, SIN, \\
& & SON, TAMS, VER, YUC, ZAC \\
\hline
\end{tabular}

Cuadro 6: Riqueza florística registrada para Morelos en este estudio y en trabajos previos. Bosque de coníferas (BC), bosque de encino (BE), bosque de galería (BG), bosque mesófilo de montaña (BMM), bosque tropical caducifolio (BTC), bosque de pino-encino (BPE), matorral xerófito (MX), pastizal (PZ), palmar (PAL), vegetación acuática (VA), vegetación secundaria (VS) (Rzedowski, 2006).

\begin{tabular}{|c|c|c|c|c|c|}
\hline Área de estudio y referencia & Tipo de vegetación & Familias & Géneros & Especies & $\begin{array}{l}\text { Superficie } \\
\left(\mathrm{km}^{2}\right)\end{array}$ \\
\hline En este estudio & BTC & 85 & 285 & 434 & 0.4 \\
\hline Cañón de Lobos (Soria, 1985) & BTC & 43 & 87 & 110 & 4499.8 \\
\hline $\begin{array}{l}\text { Cerros Sombrerito y Mariposas (Cerros-Tlatilpa } \\
\text { y Espejo-Serna, 1998) }\end{array}$ & BTC, BPE, VS & 89 & 244 & 368 (251 en BTC) & 8500 \\
\hline $\begin{array}{l}\text { Municipio de Amacuzac (Galindo-Becerril y } \\
\text { Fernández-Nava, 2002) }\end{array}$ & BTC, PAL, BG, BE & 100 & 289 & 490 & 125 \\
\hline Sierra de Huautla (Dorado et al., 2005) & BTC, BE, BEP, VS, PZ & 130 & 478 & 935 & 590.49 \\
\hline $\begin{array}{l}\text { Corredor Biológico del Chichinautzin (Pulido- } \\
\text { Esparza et al., 2009), monocotiledóneas }\end{array}$ & $\begin{array}{l}\text { BC, BE, BMM, BTC, MX, } \\
\text { PZ, VA }\end{array}$ & 25 & 132 & 359 (100 en BTC) & 659.01 \\
\hline $\begin{array}{l}\text { Corredor Biológico del Chichinautzin (Flores- } \\
\text { Castorena y Martínez-Alvarado, 2011) }\end{array}$ & $\begin{array}{l}\mathrm{BC}, \mathrm{BE}, \mathrm{BMM}, \mathrm{BTC}, \mathrm{MX} \\
\mathrm{PZ}, \mathrm{VA}\end{array}$ & 153 & 516 & 1265 & 657.21 \\
\hline $\begin{array}{l}\text { Barranca de Tepecapa (Hernández-Cárdenas et } \\
\text { al., 2014) }\end{array}$ & BTC, BMM, BPE & 99 & 266 & 434 (98 en BTC) & 3.5 \\
\hline $\begin{array}{l}\text { Parque Nacional El Tepozteco (Block y Meave, } \\
\text { 2015) }\end{array}$ & $\mathrm{BE}$ & 88 & 208 & 341 & 232.68 \\
\hline
\end{tabular}


también en otras entidades del país (Cuadro 5), incluyendo aquellas colindantes con el estado, como son Guerrero y Puebla, por lo que su presencia en el área de estudio amplía su rango de distribución conocida.

A pesar de que la vegetación del área aledaña a la zona arqueológica de Chalcatzingo ha sido notablemente modificada a lo largo de los años por los asentamientos de las comunidades prehispánicas, coloniales y contemporáneas que han habitado el lugar (Bugé, 1987; Grove, 1987), los cerros La Cantera y Delgado presentan una buena riqueza específica (434 especies), debido tal vez a que se encuentran ubicados dentro de un área protegida por el INAH (Instituto Nacional de Antropología e Historia), que ha funcionado como refugio para la diversidad de plantas vasculares, por lo que sería importante establecer un plan de manejo y conservación para evitar su deterioro o pérdida, sobre todo porque suma la importancia biológica y cultural en un mismo sitio.

\section{Conclusiones}

A pesar de que la zona arqueológica de Chalcatzingo abarca sólo 40 ha, alberga $42.7 \%$ de las familias, $26.6 \%$ de los géneros y $12.4 \%$ de las especies reportadas por Villaseñor (2016) para Morelos, lo que refleja la importancia del sitio. Además, el presente estudio pone de manifiesto la necesidad de continuar con el trabajo de exploración botánica en esta parte del estado, sobre todo en aquellas áreas de difícil acceso, ya que por sus características topográficas generalmente tienen poco o nulo valor para las actividades agropecuarias, lo que permite que se mantengan como remanentes de la diversidad biológica.

\section{Contribución de autores}

RCT, JCR, AMR, AFM y MJS concibieron y diseñaron el estudio. Todos los autores realizaron las colectas, la identificación de los ejemplares y la elaboración del manuscrito. RCT y AMR confeccionaron las figuras. Todos los autores contribuyeron con la revisión, discusión y aprobación del manuscrito final.

\section{Financiamiento}

Este estudio fue financiado con recursos personales de todos los autores.

\section{Agradecimientos}

Se agradece a las autoridades del Instituto Nacional de Antropología del estado de Morelos por las facilidades prestadas durante la realización del estudio, así como a Aarón Cortés, Andres Robert, Luis Gerardo Ávila, Mónica MiguelVázquez, Berenice López, Luisa Rodríguez, Yaneli Montoya, Norma Rueda y al señor Andrés Barranco por su valioso apoyo en el trabajo de campo. A María Luisa Alquicira y Mauricio Mendoza Flores por las fotografías con dron y a Esmeralda Urzúa por la elaboración del mapa del área de estudio. Se agradece a los revisores anónimos los comentarios y sugerencias que contribuyeron a mejorar la calidad del manuscrito.

\section{Literatura citada}

ABACo A.C. 2015. FAMEX: Clave Taxonómica para familias de plantas con flores (Magnoliophyta) de México. http:// www.abatax.abacoac.org (consultado noviembre de 2015).

APG III. 2009. An update of the Angiosperm Phylogeny Group classification for the orders and families of flowering plants: APG III. Botanical Journal of the Linnean Society 161(2): 105121. DOI: https://doi.org/10.1111/j.1095-8339.2009.00996.x

APG IV. 2016. An update of the Angiosperm Phylogeny Group classification for the orders and families of flowering plants: APG IV. Botanical Journal of the Linnean Society 181(1): 1-20. DOI: https://doi.org/10.1111/boj.12385

Block, S. y J. A. Meave. 2015. Structure and diversity of oak forests in the Tepozteco National Park (Morelos, México). Botanical Sciences 93(3): 429-460. DOI: http://dx.doi.org/10.17129/ botsci.150

Bonilla-Barbosa, J. y J. L. Villaseñor. 2003. Catálogo de la flora del estado de Morelos. Universidad Autónoma del Estado de Morelos. Cuernavaca, México. 129 pp.

Boyas, D. J., S. M. Cervantes, G. J. Javelly, A. M. Linares, A. F. Solares, E. R. Soto, T. I. Naufal, y C. L. Sandoval. 2001. Diagnóstico forestal del estado de Morelos. Instituto Nacional de Investigaciones Forestales, Agrícolas y Pecuarias. Morelos, México. 180 pp.

Bugé, D. 1987. Contemporary Agriculture at Chalcatzingo. In: Grove, D. (ed.). Ancient Chalcatzingo. University of Texas Press. Austin, USA. Pp. 409-419.

Calderón de Rzedowski, G. 1992. Loasaceae. Flora del Bajío y de Regiones Adyacentes 7: 1-26. 
Cerros-Tlatilpa, R. y A. Espejo-Serna. 1998. Contribución al estudio florístico de los cerros El Sombrerito y Las Mariposas (Zoapapalotl) en el municipio de Tlayacapan, Morelos, México. Polibotánica 8: 29-46.

Dorado, O., J. de Jesús Almonte, K. López, F. Ramos y D. Arias. 2015. Vegetación arbórea como Indicador Ambiental. In: Ortiz, M. L., E. Sánchez, M. Castrejón y M. Romero (eds.). Los indicadores ambientales como herramienta para la sustentabilidad. Estudio de caso en Morelos. Universidad Autónoma del Estado de Morelos. Cuernavaca, México. Pp. 27-54.

Dorado, O., B. Maldonado, D. Arias, V. Sorani, R. Ramírez, E. Leyva y D. Valenzuela. 2005. Programa de Conservación y Manejo de la Reserva de la Biósfera Sierra de Huautla. Comisión Nacional de Áreas Naturales Protegidas. México, D.F., México. 210 pp.

Espejo-Serna, A., J. García, A. López Ferrari, R. Jiménez Machorro y L. Sánchez Saldaña. 2002. Orquídeas del Estado de Morelos. Orquídea (México) 16: 1-392.

Espinosa-García, F., J. L. Villaseñor y H. Vibrans. 2009. Biodiversity, distribution, and possible impacts of exotic weeds in Mexico. In: van Devender, T., F. J. Espinosa-García, B. L. Harper-Lore y T. Hubbard (eds.). Invasive plants on the move. Controlling them in North America. Arizona-Sonora Desert Museum. Tucson, USA. Pp. 43-52.

FNA . 1993+. Flora of North America North of Mexico. 20+ vols. Oxford. University Press. New York, USA.

Flores-Castorena, A. y D. Martínez-Alvarado. 2011. Sinopsis Florística. In: Bonilla-Barbosa, J., M. V. Mora, J. LunaFigueroa, H. Colín y S. Santillán-Alarcón (eds.). Biodiversidad, conservación y manejo en el Corredor Biológico Chichinautzin condiciones actuales y perspectivas. Universidad Autónoma del Estado de Morelos. Morelos, México. Pp. 69-97.

Galindo-Becerril, G. y R. Fernández-Nava. 2002. Inventario florístico del municipio de Amacuzac, Morelos, México. Polibotánica 13: 107-135.

Germán, M. T. 1986. Estructura y organización del herbario. In: Lot, A. y F. Chiang (eds.). Manual de herbario. Administración y manejo de colecciones, técnicas de recolección y preparación de ejemplares botánicos. Consejo Nacional de la Flora de México. México, D.F., México. Pp. 11-30.

González-Rocha, E. y R. Cerros-Tlatilpa. 2015. La familia Apocynaceae (Apocynoideae y Rauvolfioideae) en el estado de Morelos, México. Acta Botanica Mexicana 110: 21-70. DOI: https://doi.org/10.21829/abm110.2015.191
González-Rocha, E., R. Cerros-Tlatilpa, A. Espejo-Serna, y A. R. López-Ferrari. 2015. Tillandsia chalcatzinguensis, a new species from the state of Morelos. Phytotaxa 227(2): 182188. DOI: https://dx.doi.org/10.11646/phytotaxa.227.2.8 Grove, D. C. 1987. Ancient Chalcatzingo. University of Texas Press. Austin, USA. 571 pp.

Grove, D. C. 2008. Chalcatzingo: A brief introduction. The PreColumbian Art Research Institute Journal 9: 1-7.

Guerrero, J. A., R. Cerros-Tlatilpa, E. Urzúa y A. Rizo-Aguilar. 2015. Indicadores de biodiversidad en el estado de Morelos: Situación Actual. In: Ortiz, M. L., E. Sánchez, M. L. Castrejón y M. Romero (eds). Los indicadores ambientales como herramienta para la sustentabilidad. Estudio de caso en Morelos. Universidad Autónoma del Estado de Morelos. Cuernavaca, México. Pp. 57-90.

Hernández-Cárdenas, R. A., R. Cerros-Tlatilpa y A. FloresMorales. 2014. Las plantas vasculares y vegetación de la Barranca Tepecapa en el municipio de Tlayacapan, Morelos, México. Acta Botanica Mexicana 108: 11-38. DOI: https://doi.org/10.21829/abm108.2014.200

Hernández-Cárdenas, R. A., A. E. Espejo-Serna y A. R. LópezFerrari. 2018. Revisión taxonómica del género Viridantha (Tillandsia subgénero Viridantha sensu stricto. Revista Mexicana de Biodiversidad 89(4): 1012-1032. DOI: https://dx.doi.org/10.22201/ib.20078706e.2018.4.2453

Illescas, S. J. F. y G. Buitrago. 2015. Chalcatzingo, México: donde las piedras hablan. Intervención 6: 43-53. DOI: https:// doi.org/10.30763/Intervencion.2015.11.134

INEGI. 2009. Prontuario de información geográfica municipal de los Estados Unidos Mexicanos. Instituto Nacional de Estadística y Geografía. Aguascalientes, México. Pp. 9.

INEGI. 2010. Anuario estadístico de los Estados Unidos Mexicanos, Instituto Nacional de Estadística y Geografía. Aguascalientes, México. 165 pp.

INEGI. 2016. Instituto Nacional de Estadística y Geografía, Mapa de Suelos. http://www.beta.inegi.org.mx/temas/ mapas/edafologia/ (consultado abril de 2016).

JSTOR. 2019. JSTOR Global Plants. http://plants.jstor.org/ (consultado marzo de 2019).

Miguel-Vázquez, M. I. y R. Cerros-Tlatilpa. 2013. Onagraceae de Morelos, México. Revista Mexicana de Biodiversidad 84(4): 1309-1315. DOI: https://doi.org/10.7550/ rmb. 24854 
Milliken, W., B. Klitgård y A. Baracat, A. 2009+. Neotropikey Interactive key and information resources for flowering plants of the Neotropics. http://www.kew.org/science/tropamerica/ neotropikey.htm (consultado noviembre de 2017).

MNHN. 2018. Muséum National d’Histoire Naturelle. https:// science.mnhn.fr/institution/mnhn/collection/p/ (consultado diciembre de 2018).

NYBG. 2018. The New York Botanical Garden, C.V. Starr Virtual Herbarium. http://sweetgum.nybg.org/science/vh/ (consultado diciembre de 2018).

Pérez-Calix, E. 2008. Crassulaceae. Flora del Bajío y de Regiones Adyacentes 156: 1-141.

PPG I. 2016. A community-derived classification for extant lycophytes and ferns. Journal of Systematics and Evolution 54(6): 563-603. DOI: https://doi.org/10.1111/jse.12229

Pulido-Esparza, V. A., A. Espejo-Serna y A. R. López-Ferrari. 2009. Las monocotiledóneas nativas del Corredor Biológico Chichinautzin. Acta Botanica Mexicana 86: 9-38. DOI: https://doi.org/10.21829/abm86.2009.1076

Ramírez, R. R. y O. Téllez. 1992. Las Dioscóreas (Dioscoreaceae) del estado de Morelos, Mexico. Anales del Instituto de Biología, Serie Botánica 63: 67-99.

RBGK, 2019. The Herbarium Catalogue. Royal Botanic Gardens, Kew. http://www.kew.org/herbcat (consultado febrero de 2019).

Riba, R., L. Pacheco, A. Valdés. y Y. Sandoval. 1996. Pteridoflora del estado de Morelos, México. Lista de familias, géneros y especies. Acta Botanica Mexicana 37: 45-65. DOI: https:// doi.org/10.21829/abm37.1996.769

Rzedowski, J. 1991. El endemismo en la flora fanerogámica mexicana: Una apreciación analítica preliminar. Acta Botanica Mexicana 15: 47-64. DOI: https://doi. org/10.21829/abm15.1991.620

Rzedowski, J. 2006. Vegetación de México, edición digital. Ciudad de México. Comisión Nacional para el Conocimiento y Uso de la Biodiversidad. www.biodiversidad.gob.mx/ publicaciones/librosDig/pdf/VegetacionMx_Cont.pdf (consultado abril de 2016).

Rzedowski, J. y G. Calderón de Rzedowski. 2013. Datos para la apreciación de la flora fanerógama del bosque tropical caducifolio de México. Acta Botanica Mexicana 102: 1-23. DOI: https://doi.org/10.21829/abm102.2013.229

Sánchez-Ken, J. G. y R. Cerros-Tlatilpa. 2016. Listado florístico de la familia Poaceae del estado de Morelos, México.
Acta Botanica Mexicana 116: 65-105. DOI: https://doi. org/10.21829/abm116.2016.119

Sánchez-Ken, J. G., G. A. Zita-Padilla y M. Mendoza-Cruz. 2012. Catálogo de malezas gramíneas nativas e introducidas de México. Consejo Nacional Consultivo Fitosanitario. México, D.F., México. 433 pp.

SEMARNAT. 2010. NORMA Oficial Mexicana NOM-059SEMARNAT-2010, Protección ambiental-Especies nativas de México de Flora y Fauna Silvestres-Categorías de Riesgo y especificaciones para su inclusión, exclusión o cambio-Lista de Especies en Riesgo. Secretaría del Medio Ambiente y Recursos Naturales. Diario Oficial dela Federación.Cd. Mx., México. http:// dof.gob.mx/nota_detalle_popup.php?codigo=30/12/2010.

SNMNH. 2018. National Museum of Natural History. The United States National Herbarium. https://collections.nmnh. si.edu/search/botany/ (consultado mayo de 2018).

Soria, R. G. 1985. Flora de Morelos: Descripción de especies vegetales de la Selva Baja Caducifolia del Cañón de Lobos, municipio de Yautepec. Universidad Autónoma del Estado de Morelos. Cuernavaca, México. 163 pp.

Suárez-Mota, M. E., O. Téllez-Valdés, R. Lira-Saade y J. Villaseñor. 2013. Una regionalización de la Faja Volcánica Transmexicana con base en su riqueza florística. Botanical Sciences 91(1): 93-105. DOI: https://dx.doi.org/10.17129/botsci.405

Trejo, I. y R. Dirzo. 2000. Deforestation in seasonally dry tropical forest: A national and local analysis in Mexico. Biological Conservation 94(2): 133-142. DOI: https://doi.org/10.1016/ S0006-3207(99)00188-3

Trejo, I. y R. Dirzo. 2002. Floristic diversity of Mexican seasonally dry tropical forests. Biodiversity and Conservation 11(11): 20632084. DOI: https://doi.org/10.1023/A:1020876316013

TROPICOS. 2019. Tropicos.org. Missouri Botanical Garden. http:// www.tropicos.org (consultado marzo de 2019).

Tucker, G. C. 1994. Revision of the Mexican species of Cyperus (Cyperaceae). Systematic Botany Monographs 43: 1-213. DOI: https://doi.org/10.2307/25027842

Vázquez, S. J. 1974. Contribución al estudio de las plantas del estado de Morelos (México), Catálogo de las plantas contenidas en el “Herbario L’Amagatall”. Ciencia 29: 1-138.

Vázquez-Sánchez, M, T. Terrazas y S. Arias. 2012. El hábito y la forma de crecimiento en la Tribu Cacteae (Cactaceae, Cactoideae). Botanical Sciences 90(2): 97-108. DOI: https:// doi.org/10.17129/botsci.477 
Villaseñor, J. L. 2003. Diversidad y distribución de las Magnoliophyta de México. Interciencia 28: 160-167.

Villaseñor, J. L. 2016. Checklist of the native vascular plants of Mexico. Revista Mexicana de Biodiversidad 87(3): 559-902. DOI: https://doi.org/10.1016/j.rmb.2016.06.017
Villaseñor, J. L. y F. Espinosa-García. 2004. The alien flowering plants of Mexico. Diversity and Distributions 10(2): 113-123. DOI: https://doi.org/10.1111/j.1366-9516.2004.00059.x 
Apéndice: Plantas vasculares de los cerros Delgado y La Cantera, Jantetelco, Morelos, México, ordenadas alfabéticamente por familia con los nombres aceptados por el sistema APG III (2009), APG IV (2016) y PPG I (2016). Los símbolos indican: especies endémicas de México (*), especies endémicas de Morelos ( $\boldsymbol{\nabla})$, nuevos registros de Morelos (•), especies registradas en la NOM-ECOL-059-SEMARNAT-2010 ( $\diamond)$. Los ejemplares colectados se depositaron en los herbarios HUAP, HUMO, MEXU y UAMIZ. Recolectores: Rosa Cerros Tlatilpa (RCT), Adolfo Espejo Serna (AES), Alejandro Flores Morales (AFM), Edith González Rocha (EGR), Gerald Matus Hernández Barón (GMHB), Rodrigo Alejandro Hernández Cárdenas (RAHC), Mayo Jaramillo Sánchez (MJS), Aniceto Mendoza Ramírez (AMR), Mónica Isabel Miguel Vázquez (MIMV), Yaneli Montoya Molina (YMM), Luisa Olivia Morales Rodríguez (LORM). Formas de crecimiento: acuática (Ac), árbol (A), candelabriforme (Can), arbusto (Ar), cilíndrica (Ci), epífita (E), globosa $(G)$, trepadora ( $T$, incluye herbáceas y leñosas), hierba ( $H$, incluye sufrútices), parásita (P). Especie nativa (Na), especie exótica (Ex). La forma de crecimiento para la familia Cactaceae se basó en Vázquez-Sánchez et al. (2012).

\begin{tabular}{|c|c|c|c|}
\hline Familias/especies & Ejemplares examinados & $\begin{array}{l}\text { Formas de } \\
\text { crecimiento }\end{array}$ & $\begin{array}{r}\text { Exótica/ } \\
\text { Nativa }\end{array}$ \\
\hline
\end{tabular}

\section{PTERIDOFITAS Y AFINES}

Anemiaceae

Anemia hirsuta (L.) Sw.

AMR-2106 (UAMIZ 81794), GMHB-309 (HUMO 36481), RCT-3033 (HUMO 36626)

Anemia tomentosa (Savigny) Sw. var. mexicana (C. Presl) Mickel

AMR-2081 (UAMIZ 81809), GMHB-152 (HUMO 36831), GMHB-164B (HUMO 36844), GMHB-253 (HUMO 36648)

Aspleniaceae

Asplenium pumilum Sw.

AMR-2351 (UAMIZ 84846)

AFM-379 (HUAP 75858)

ex Fée) Maxon

\section{Dryopteridaceae}

Dryopteris karwinskyana (Mett.) Kuntze

\section{Ophioglossaceae}

Ophioglossum nudicaule L. f.

\section{Pteridaceae}

Adiantum capillus-veneris $\mathrm{L}$.

Adiantum concinnum Humb. \& Bonpl. ex Willd.

Astrolepis sinuata (Lag. ex Sw.) D.M. Benham \&

Windham

Bommeria pedata (Sw.) E. Fourn.

Cheilanthes brachypus (Kunze) Kunze

Cheilanthes lozanoi (Maxon) R.M. Tryon \& A.F. Tryon var. seemannii (Hook.) Mickel \& Beitel *

Cheilanthes skinneri (Hook.) T. Moore

Gaga chaerophylla (M. Martens \& Galeotti) F.W. Li \& Windham

Gaga kaulfussii (Kunze) F.W. Li \& Windham

Myriopteris allosuroides (Mett.) Grusz \& Windham

*

Myriopteris aurea (Poir.) Grusz \& Windham

Myriopteris myriophylla (Desv.) Sm.
AMR-2102 (UAMIZ 81796), GMHB-185 (HUMO 36792)

AMR-2086 (UAMIZ 84845), GMHB-197 (HUMO 36560)

AMR-2077 (UAMIZ 81812)

AMR-2353 (UAMIZ 84847)

AMR-2158 (UAMIZ 80001)

AMR-2083 (UAMIZ 81810)

AMR-2157 (UAMIZ 80002), GMHB-151 (HUMO 36871),

GMHB-203 (HUMO 36631), GMHB-264A (HUMO 36800), GMHB-369 (HUMO 36645)

AMR-2353 (UAMIZ 81805), GMHB-195 (HUMO 36561), GMHB-279A (HUMO 36771)

AMR-2107B (UAMIZ81793), GMHB-205 (HUMO 36630), GMHB-289 (HUMO 36661)

AMR-2101 (UAMIZ 81798), GMHB-280 (HUMO 36784)

AMR-2153 (UAMIZ 79997)

AMR-2098 (UAMIZ 81799)

AMR-2096 (UAMIZ 81802), GMHB 260 (HUMO 36798),

MJS-16 (HUMO 37616)

AMR-2155 (UAMIZ 80003)
$\mathrm{H}$

$\mathrm{H}$

$\mathrm{H}$

$\mathrm{H}$

$\mathrm{Na}$

$\mathrm{Na}$

$\mathrm{Na}$

$\mathrm{Na}$

$\mathrm{Na}$

$\mathrm{H}$

$\mathrm{Na}$

$\mathrm{H}$

$\mathrm{Na}$

$\mathrm{H}$

$\mathrm{Na}$

$\mathrm{H}$

$\mathrm{Na}$

H

$\mathrm{Na}$

$\mathrm{H}$

$\mathrm{Na}$

$\mathrm{H}$

$\mathrm{Na}$

$\mathrm{H}$

$\mathrm{Na}$

H

$\mathrm{Na}$

$\mathrm{H}$

$\mathrm{Na}$

H

$\mathrm{Na}$

H

$\mathrm{Na}$

$\mathrm{H}$

$\mathrm{Na}$ 
Apéndice: Continuación.

\begin{tabular}{|c|c|c|c|}
\hline Familias/especies & Ejemplares examinados & $\begin{array}{l}\text { Formas de } \\
\text { crecimiento }\end{array}$ & $\begin{array}{r}\text { Exótica/ } \\
\text { Nativa }\end{array}$ \\
\hline $\begin{array}{l}\text { Notholaena lemmonii D.C. Eaton var. australis R.M. } \\
\text { Tryon • }\end{array}$ & AMR-2154 (UAMIZ 79999), GMHB-252 (HUMO 36648) & $\mathrm{H}$ & $\mathrm{Na}$ \\
\hline Pellaea oaxacana Mickel \& Beitel * & AMR-2060 (UAMIZ 80000) & $\mathrm{H}$ & $\mathrm{Na}$ \\
\hline \multicolumn{4}{|l|}{ Selaginellaceae } \\
\hline Selaginella delicatissima Linden ex A. Braun & $\begin{array}{l}\text { AMR-2084 (UAMIZ 81808), GMHB-113 (HUMO 36992), } \\
\text { GMHB-124 (HUMO 36991), GMHB-154 (HUMO 36870) }\end{array}$ & $\mathrm{H}$ & $\mathrm{Na}$ \\
\hline Selaginella lepidophylla (Hook. \& Grev.) Spring & AMR-2087 (UAMIZ 81806), GMHB-94A (HUMO 36384) & $\mathrm{H}$ & $\mathrm{Na}$ \\
\hline Selaginella pallescens (C. Presl) Spring & $\begin{array}{l}\text { AMR-2085 (UAMIZ 81807), GMHB-153 (HUMO 36868), } \\
\text { GMHB-284 (HUMO 36793), GMHB-283 (HUMO 37624) }\end{array}$ & $\mathrm{H}$ & $\mathrm{Na}$ \\
\hline Selaginella sartorii Hieron. & $\begin{array}{l}\text { AMR-2156 (UAMIZ 79998), GMHB-97 (HUMO 36426), } \\
\text { GMHB-349 (HUMO 36879) }\end{array}$ & $\mathrm{H}$ & $\mathrm{Na}$ \\
\hline \multicolumn{4}{|l|}{ Thelypteridaceae } \\
\hline Thelypteris kunthii (Desv.) C.V. Morton & AMR-2078 (UAMIZ 81813), GMHB-76 (HUMO 36444) & $\mathrm{H}$ & $\mathrm{Na}$ \\
\hline \multicolumn{4}{|l|}{ MONOCOTILEDÓNEAS } \\
\hline \multicolumn{4}{|l|}{ Amaryllidaceae } \\
\hline Hymenocallis graminifolia Greenm. * & GMHB-162 (HUMO 36668, HUAP 75528) & $\mathrm{H}$ & $\mathrm{Na}$ \\
\hline Sprekelia formosissima (L.) Herb. * & AFM-375 (HUMO 36717), GMHB-108 (HUMO 37020) & $\mathrm{H}$ & $\mathrm{Na}$ \\
\hline Zephyranthes sp. & GMHB-93 (HUMO 36458) & $\mathrm{H}$ & - \\
\hline \multicolumn{4}{|l|}{ Araceae } \\
\hline Lemna minuta Kunth & GMHB-75 (HUMO 36402) & Ac & $\mathrm{Na}$ \\
\hline Lemna obscura (Austin) Daubs & MJS-7 (HUMO 37607) & Ac & $\mathrm{Na}$ \\
\hline \multicolumn{4}{|l|}{ Asparagaceae } \\
\hline Agave angustiarum Trel. * & GMHB-38 (HUMO 36540) & $\mathrm{H}$ & $\mathrm{Na}$ \\
\hline Agave angustifolia Haw. & GMHB-155 (HUMO 36869, HUAP 75520) & $\mathrm{H}$ & $\mathrm{Na}$ \\
\hline Bessera elegans Schult. f. ** & GMHB-191 (HUMO 36565) & $\mathrm{H}$ & $\mathrm{Na}$ \\
\hline $\begin{array}{l}\text { Echeandia albiflora (Schltdl. \& Cham.) M. Martens } \\
\text { \& Galeotti米 }\end{array}$ & $\begin{array}{l}\text { GMHB-220 (HUMO 36683), GMHB-244 (HUMO 36658, } \\
\text { HUAP 75586) }\end{array}$ & $\mathrm{H}$ & $\mathrm{Na}$ \\
\hline Manfreda scabra (Ortega) McVaugh & GMHB-120C (HUMO 37021), GMHB-255 (HUMO 36555) & $\mathrm{H}$ & $\mathrm{Na}$ \\
\hline Milla biflora Cav. & $\begin{array}{l}\text { GMHB-136 (HUMO 37019, HUAP 75514), GMHB-256B } \\
\text { (HUMO 36486) }\end{array}$ & H & $\mathrm{Na}$ \\
\hline \multicolumn{4}{|l|}{ Bromeliaceae } \\
\hline $\begin{array}{l}\text { Hechtia caulescens López-Ferr., Espejo \& Mart.- } \\
\text { Correa* }\end{array}$ & AES-5890 (UAMIZ 55890), GMHB-230A (HUMO 36684) & $\mathrm{H}$ & $\mathrm{Na}$ \\
\hline Pitcairnia karwinskyana Schult. \& Schult. f. & GMHB-110 (UAMIZ 85114) & $\mathrm{H}$ & $\mathrm{Na}$ \\
\hline Tillandsia achyrostachys E. Morren ex Baker* & $\begin{array}{l}\text { AFM-403 (HUMO 36714), EGR-214 (UAMIZ 78293), } \\
\text { GMHB-81 (HUMO 36424) }\end{array}$ & $\mathrm{E}$ & $\mathrm{Na}$ \\
\hline Tillandsia caput-medusae E. Morren & GMHB-42 (HUMO 36470, HUAP 75444) & $\mathrm{E}$ & $\mathrm{Na}$ \\
\hline \multirow[t]{2}{*}{$\begin{array}{l}\text { Tillandsia chalcatzingensis Gonz.-Rocha, Cerros, } \\
\text { López-Ferr. \& Espejo } * \text { - }\end{array}$} & RCT-2969 (UAMIZ 79512) & $\mathrm{H}$ & $\mathrm{Na}$ \\
\hline & AES-5889 (UAMIZ 43054), EGR-103 (UAMIZ 78306), & & \\
\hline Tillandsia circinnatioides Matuda* & $\begin{array}{l}\text { GMHB-10 (HUAP 75463), GMHB-83 (HUMO 36390, } \\
\text { HUAP 75477) }\end{array}$ & $\mathrm{E}$ & $\mathrm{Na}$ \\
\hline Tillandsia grandispica Ehlers $*$ & AMR-2159 (UAMIZ79341) & $\mathrm{E}$ & $\mathrm{Na}$ \\
\hline
\end{tabular}


Apéndice: Continuación.

\begin{tabular}{|c|c|c|c|}
\hline Familias/especies & Ejemplares examinados & $\begin{array}{l}\text { Formas de } \\
\text { crecimiento }\end{array}$ & $\begin{array}{r}\text { Exótica/ } \\
\text { Nativa }\end{array}$ \\
\hline Tillandsia makoyana Baker & GMHB-82 (HUMO 36423) & $\mathrm{E}$ & $\mathrm{Na}$ \\
\hline Tillandsia recurvata (L.) L. & $\begin{array}{l}\text { AFM-319 (HUMO 36583, HUAP 75821), AFM-404 (HUMO } \\
\text { 36718), GMHB-16 (HUMO 36804) }\end{array}$ & $\mathrm{E}$ & $\mathrm{Na}$ \\
\hline $\begin{array}{l}\text { Viridantha rzedowskiana Hern.-Cárdenas, Espejo \& } \\
\text { López-Ferr. } * \text { - }\end{array}$ & GMHB-41 (HUMO 37645), RAHC-2108 (UAMIZ 84461) & $\mathrm{H}$ & $\mathrm{Na}$ \\
\hline \multicolumn{4}{|l|}{ Commelinaceae } \\
\hline Commelina dianthifolia Delile & $\begin{array}{l}\text { AMR-2094 (UAMIZ 79990) } \\
\text { AMR-2091 (UAMIZ 79994), GMHB-78 (HUMO 36454), }\end{array}$ & $\mathrm{H}$ & $\mathrm{Na}$ \\
\hline Commelina diffusa Burm. f. & $\begin{array}{l}\text { GMHB-132 (HUMO 37004), GMHB-192 (HUMO 36573), } \\
\text { MJS-6 (HUMO 37632), RCT-3009 (HUAP 75680) }\end{array}$ & $\mathrm{H}$ & $\mathrm{Na}$ \\
\hline Commelina erecta $\mathrm{L}$. & $\begin{array}{l}\text { GMHB-169 (HUMO 366671, HUAP 75532), GMHB-182A } \\
\text { (HUMO 36567, HUAP 75540) }\end{array}$ & $\mathrm{H}$ & $\mathrm{Na}$ \\
\hline $\begin{array}{l}\text { Thyrsanthemum macrophyllum (Greenm.) } \\
\text { Rohweder * }\end{array}$ & GMHB-188A (HUMO 36887, HUAP 75522) & $\mathrm{H}$ & $\mathrm{Na}$ \\
\hline Tinantia erecta (Jacq.) Schltdl. & AMR-2099 (UAMIZ 79991), GMHB-235 (HUMO 36636) & $\mathrm{H}$ & $\mathrm{Na}$ \\
\hline Tradescantia andrieuxii C.B. Clarke $*$ & MIMV-489 (HUMO 37611) & $\mathrm{H}$ & $\mathrm{Na}$ \\
\hline $\begin{array}{l}\text { Tripogandra amplexicaulis (Klotzsch ex C.B. Clarke) } \\
\text { Woodson }\end{array}$ & $\begin{array}{l}\text { GMHB-332 (HUMO 36487, HUAP 75644), RCT-3015 } \\
\text { (HUMO 36760, HUAP 75685) }\end{array}$ & $\mathrm{H}$ & $\mathrm{Na}$ \\
\hline Tripogandra angustifolia (B.L. Rob.) Woodson & $\begin{array}{l}\text { GMHB-318 (HUMO 36355, HUAP 75635), MJS-5 (HUMO } \\
\text { 37633) }\end{array}$ & $\mathrm{H}$ & $\mathrm{Na}$ \\
\hline Tripogandra saxicola (Greenm.) Woodson * & $\begin{array}{l}\text { AMR-2105 (UAMIZ 79989), GMHB-302 (HUMO 36486, } \\
\text { HUAP 75625), GMHB-367 (HUMO 36818) }\end{array}$ & $\mathrm{H}$ & $\mathrm{Na}$ \\
\hline \multicolumn{4}{|l|}{ Cyperaceae } \\
\hline Cyperus amabilis Vahl $\bullet$ & $\begin{array}{l}\text { GMHB-306 (HUMO 36482, HUAP 75628), RCT-3027B } \\
\text { (HUMO 36578, HUAP 75696), YMM- } 292 \text { (HUMO 36929, } \\
\text { HUAP 75809) }\end{array}$ & $\mathrm{H}$ & $\mathrm{Na}$ \\
\hline Cyperus esculentus L. & $\begin{array}{l}\text { GMHB-184 HUMO (36566, HUAP 75543) } \\
\text { GMHB-98 (HUMO 36459, HUAP 75490), GMHB-182B }\end{array}$ & $\mathrm{H}$ & Ex \\
\hline Cyperus seslerioides Kunth & $\begin{array}{l}\text { (HUMO 36832), GMHB-295 (HUMO 36492, HUAP } \\
\text { 75621) }\end{array}$ & $\mathrm{H}$ & $\mathrm{Na}$ \\
\hline Cyperus squarrosus L. & GMHB-240 (HUMO 36639, HUAP 75584) & $\mathrm{H}$ & $\mathrm{Na}$ \\
\hline Eleocharis acicularis (L.) Roem. \& Schult. & RCT-3024 (HUMO 36737, HUAP 75692) & $\mathrm{H}$ & $\mathrm{Na}$ \\
\hline Eleocharis montevidensis Kunth & $\begin{array}{l}\text { GMHB-198 (HUMO 36559, HUAP 75553), GMHB-251 } \\
\text { (HUMO 36649, HUAP 75593) }\end{array}$ & $\mathrm{H}$ & $\mathrm{Na}$ \\
\hline Lipocarpha micrantha (Vahl) G.C. Tucker & GMHB-368 (HUMO 36830) & $\mathrm{H}$ & $\mathrm{Na}$ \\
\hline Rhynchospora cf. brevirostris Griseb. & RCT-3027A (HUMO 36736, HUAP 75695) & $\mathrm{H}$ & $\mathrm{Na}$ \\
\hline \multicolumn{4}{|l|}{ Iridaceae } \\
\hline Sisyrinchium tenuifolium Humb. \& Bonpl. ex Willd. & GHMB-142 (HUMO 36986) & $\mathrm{H}$ & $\mathrm{Na}$ \\
\hline Sisyrinchium tolucense Peyr. * & $\begin{array}{l}\text { AMR-2089 (UAMIZ 76494), GMHB-179 (HUMO 36569, } \\
\text { HUAP 75538) }\end{array}$ & $\mathrm{H}$ & $\mathrm{Na}$ \\
\hline \multicolumn{4}{|l|}{ Marantaceae } \\
\hline Maranta arundinacea $\mathrm{L}$. & GMHB-187 (HUMO 36553, HUAP 75545) & $\mathrm{H}$ & $\mathrm{Na}$ \\
\hline
\end{tabular}


Apéndice: Continuación.

\begin{tabular}{|c|c|c|c|}
\hline Familias/especies & Ejemplares examinados & $\begin{array}{l}\text { Formas de } \\
\text { crecimiento }\end{array}$ & $\begin{array}{r}\text { Exótica/ } \\
\text { Nativa }\end{array}$ \\
\hline \multicolumn{4}{|l|}{ Orchidaceae } \\
\hline Aulosepalum tenuiflorum (Greenm.) Garay* & GMHB-34 (HUMO 36499) & $\mathrm{H}$ & $\mathrm{Na}$ \\
\hline Bletia coccinea Lex. * & GMHB-201 (HUMO 36629) & $\mathrm{H}$ & $\mathrm{Na}$ \\
\hline $\begin{array}{l}\text { Cyrtopodium macrobulbon (Lex.) G.A. Romero \& } \\
\text { Carnevali }\end{array}$ & GMHB-58 (HUMO 36456, HUAP 75454) & $\mathrm{H}$ & $\mathrm{Na}$ \\
\hline $\begin{array}{l}\text { Dichromanthus michuacanus (Lex.) Salazar \& Soto } \\
\text { Arenas }\end{array}$ & AES-5891 (UAMIZ 42903) & $\mathrm{H}$ & $\mathrm{Na}$ \\
\hline Encyclia spatella (Rchb. f.) Schltr. ** & AFM-410 (HUMO 36713, HUAP 75870) & $\mathrm{H}$ & $\mathrm{Na}$ \\
\hline Epidendrum ciliare L. & AFM-sn (HUMO 37603) & $\mathrm{H}$ & $\mathrm{Na}$ \\
\hline \multicolumn{4}{|l|}{ Poaceae } \\
\hline Aristida adscensionis $\mathrm{L}$. & $\begin{array}{l}\text { GMHB-256A (HUMO 36802), YMM-301 (HUMO 36901, } \\
\text { HUAP 75815) }\end{array}$ & $\mathrm{H}$ & $\mathrm{Na}$ \\
\hline Aristida ternipes Cav. & $\begin{array}{l}\text { MJS-15 (HUMO 37653), YMM-306 (HUMO 36872, HUAP } \\
\text { 75766) }\end{array}$ & $\mathrm{H}$ & $\mathrm{Na}$ \\
\hline Arundinella deppeana Nees & AFM-362 (HUMO 36629, HUAP 75852) & $\mathrm{H}$ & $\mathrm{Na}$ \\
\hline Bothriochloa pertusa (L.) A. Camus & YMM-288 (HUMO 36924, HUAP 75807) & $\mathrm{H}$ & Ex \\
\hline Bouteloua barbata Lag. & YMM-294 (HUMO 36896, HUAP 75810) & $\mathrm{H}$ & $\mathrm{Na}$ \\
\hline $\begin{array}{l}\text { Bouteloua curtipendula (Michx.) Torr. var. } \\
\text { curtipendula }\end{array}$ & GMHB-364 (HUMO 36874) & $\mathrm{H}$ & $\mathrm{Na}$ \\
\hline Bouteloua dimorpha Columbus & GMHB-320 (HUMO 36499), GMHB-326 (HUMO 36485) & $\mathrm{H}$ & $\mathrm{Na}$ \\
\hline Bouteloua diversispicula Columbus & GMHB-317 (HUAP 75634) & $\mathrm{H}$ & $\mathrm{Na}$ \\
\hline Bouteloua erecta (Vasey \& Hack.) Columbus & MJS-10 (HUMO 37618) & $\mathrm{H}$ & $\mathrm{Na}$ \\
\hline Bouteloua media (E. Fourn.) Gould \& Kapadia & RCT-3020B (HUMO 36733) & $\mathrm{H}$ & $\mathrm{Na}$ \\
\hline Bouteloua polymorpha (E. Fourn.) Columbus* & $\begin{array}{l}\text { GMHB-221 (HUMO 36635, HUAP 75568), GMHB-250 } \\
\text { (HUMO 36637, HUAP 75592), YMM-345 (HUMO 36822, } \\
\text { HUAP 75790) }\end{array}$ & $\mathrm{H}$ & $\mathrm{Na}$ \\
\hline Bouteloua repens (Kunth) Scribn. \& Merr. & $\begin{array}{l}\text { GMHB-223 (HUMO 36676, HUAP 75569), GMHB-239 } \\
\text { (HUMO 36642, HUAP 75583), YMM-289B (HUMO } \\
\text { 36926) }\end{array}$ & $\mathrm{H}$ & $\mathrm{Na}$ \\
\hline Cenchrus echinatus L. & YMM-354 (HUMO 36888, HUAP 75755) & $\mathrm{H}$ & $\mathrm{Na}$ \\
\hline Cenchrus pilosus Kunth & $\begin{array}{l}\text { GHMB-69 (HUMO 36436), RCT-3011 (HUMO 36749, } \\
\text { HUAP 75682), RCT-3067 (HUAP 75725), YMM-313 } \\
\text { (HUMO 36850) }\end{array}$ & $\mathrm{H}$ & $\mathrm{Na}$ \\
\hline Cenchrus polystachios (L.) Morrone & AFM-383 (HUMO 36721), RCT-3011 (HUMO 36749) & $\mathrm{H}$ & Ex \\
\hline Chloris virgata Sw. & $\begin{array}{l}\text { AFM-340 (HUAP 75835), GMHB-297 (HUMO 36791, HUAP } \\
75622 \text { ) }\end{array}$ & $\mathrm{H}$ & $\mathrm{Na}$ \\
\hline Cynodon dactylon (L.) Pers. & GMHB-71 (HUMO 36377) & $\mathrm{H}$ & Ex \\
\hline Diectomis fastigiata (Sw.) P. Beauv. & YMM-321 (HUMO 36912, HUAP 75776) & $\mathrm{H}$ & $\mathrm{Na}$ \\
\hline & AFM-328 (HUMO 36584, HUAP 75828), GMHB-224 & & \\
\hline Digitaria bicornis (Lam.) Roem. \& Schult. & $\begin{array}{l}\text { (HUMO 36685, HUAP 75570), RCT-3044 (HUAP 75709), } \\
\text { YMM } 326 \text { (HUAP 75779), YMM-345 (HUMO 36822) }\end{array}$ & $\mathrm{H}$ & Ex \\
\hline Echinochloa crus-galli (L.) P. Beauv. var. crus-galli & $\begin{array}{l}\text { AFM-329 (HUMO 36598, HUAP 75829), GMHB-68 (HUMO } \\
\text { 3636, HUAP 75472) }\end{array}$ & $\mathrm{H}$ & Ex \\
\hline
\end{tabular}


Apéndice: Continuación.

\begin{tabular}{|c|c|c|c|}
\hline Familias/especies & Ejemplares examinados & $\begin{array}{l}\text { Formas de } \\
\text { crecimiento }\end{array}$ & $\begin{array}{r}\text { Exótica/ } \\
\text { Nativa }\end{array}$ \\
\hline Eleusine indica (L.) Gaertn. & $\begin{array}{l}\text { AFM-339 (HUMO 36620), GMHB-67 (HUMO 36385, HUAP } \\
\text { 75471), YMM-351 (HUMO 36910) }\end{array}$ & $\mathrm{H}$ & Ex \\
\hline Eragrostis ciliaris (L.) R. Br. & RCT-3083 (HUAP 75735) & $\mathrm{H}$ & $\mathrm{Na}$ \\
\hline Eragrostis maypurensis (Kunth) Steud. & $\begin{array}{l}\text { RCT-3032 (HUMO 36607, HUAP 75700), YMM-328 } \\
\text { (HUMO 36825, HUAP 75781) }\end{array}$ & $\mathrm{H}$ & $\mathrm{Na}$ \\
\hline Eragrostis mexicana (Hornem.) Link var. mexicana & $\begin{array}{l}\text { GMHB-273 (HUMO 36569, HUAP 75606), GMHB-301 } \\
\text { (HUMO 36474, HUAP 75624), GMHB-303A (HUMO } \\
\text { 36471), YMM-319 (HUMO 36404) }\end{array}$ & $\mathrm{H}$ & $\mathrm{Na}$ \\
\hline $\begin{array}{l}\text { Eragrostis pectinacea (Michx.) Nees var. miserrima } \\
\text { (E. Fourn.) Reeder }\end{array}$ & GMHB-311 (HUMO 36476), YMM-307B (HUMO 36860) & $\mathrm{H}$ & $\mathrm{Na}$ \\
\hline Eragrostis pilosa (L.) P. Beauv. & GMHB-211 (HUMO 36674) & $\mathrm{H}$ & Ex \\
\hline Eragrostis viscosa (Retz.) Trin. & YMM-308 (HUMO 36941, HUAP 75767) & $\mathrm{H}$ & Ex \\
\hline Hilaria belangeri (Steud.) Nash & YMM-287 (HUMO 36923, HUAP 75806) & $\mathrm{H}$ & $\mathrm{Na}$ \\
\hline Hilaria hintonii Sohns $*$ & YMM-289A (HUMO 36925, HUAP 75808) & $\mathrm{H}$ & $\mathrm{Na}$ \\
\hline Hyperthelia dissoluta (Nees) Clayton & GMHB-248 (HUMO 36644, HUAP 75569) & $\mathrm{H}$ & Ex \\
\hline Ixophorus unisetus (J. Presl) Schltdl. & $R C T-3021$ (HUMO 36732) & $\mathrm{H}$ & $\mathrm{Na}$ \\
\hline Lasiacis nigra Davidse & RCT-3042 (HUMO 36921) & $\mathrm{H}$ & $\mathrm{Na}$ \\
\hline Lasiacis procerrima (Hack.) Hitchc. & AFM-380 (HUMO 36692, HUAP 75859) & $\mathrm{H}$ & $\mathrm{Na}$ \\
\hline Lasiacis ruscifolia (Kunth) Hitchc. var. ruscifolia & $\begin{array}{l}\text { AFM-366 (HUMO 36708, HUAP 75853), AFM-398 (HUMO } \\
\text { 26716), GMHB-26 (HUMO 36783, HUAP 75456), YMM- } \\
319 \text { (HUMO 36404), GMHB-236B (HUMO 37625) }\end{array}$ & $\mathrm{H}$ & $\mathrm{Na}$ \\
\hline Melinis repens (Willd.) Zizka & $\begin{array}{l}\text { AFM-386 (HUMO 37657, HUAP 75863), YMM-323 (HUMO } \\
\text { 36932) }\end{array}$ & $\mathrm{H}$ & Ex \\
\hline Microchloa kunthii Desv. & GMHB-140 (HUMO 37006, HUAP 75516) & $\mathrm{H}$ & $\mathrm{Na}$ \\
\hline Muhlenbergia implicata (Kunth) Trin. & YMM-323 (HUMO 36932, HUAP 75777) & $\mathrm{H}$ & $\mathrm{Na}$ \\
\hline Muhlenbergia pectinata C.O. Godd. & YMM-348 (HUMO 36947, HUAP 75791) & $\mathrm{H}$ & $\mathrm{Na}$ \\
\hline Muhlenbergia tenella (Kunth) Trin. & $\begin{array}{l}\text { RCT-3013 (HUMO 36739, HUAP 75684), YMM-276 } \\
\text { (HUMO 36917, HUAP 75803) }\end{array}$ & $\mathrm{H}$ & $\mathrm{Na}$ \\
\hline Muhlenbergia uniseta (Lag.) Columbus* & YMM-324 (HUMO 36931) & $\mathrm{H}$ & $\mathrm{Na}$ \\
\hline $\begin{array}{l}\text { Oplismenus burmannii (Retz.) P. Beauv. var. } \\
\text { burmannii }\end{array}$ & GMHB-264B (HUMO 36782), YMM-322 (HUMO 36877) & $\mathrm{H}$ & $\mathrm{Na}$ \\
\hline Panicum parcum Hitchc. \& Chase & $\begin{array}{l}\text { GMHB-257 (HUMO 36558), GMHB-266 (HUMO 36557, } \\
\text { HUMO 36773, HUAP 75601) }\end{array}$ & $\mathrm{H}$ & $\mathrm{Na}$ \\
\hline Paspalum convexum Humb. \& Bonpl. ex Flüggé & $\begin{array}{l}\text { GMHB-210 (HUMO 366677, HUAP 75559), GMHB-245 } \\
\text { (HUMO 36662, HUAP 75587), YMM-341 (HUMO 36856, } \\
\text { HUAP 75792) }\end{array}$ & $\mathrm{H}$ & $\mathrm{Na}$ \\
\hline Paspalum unispicatum (Scribn. \& Merr.) Nash & GMHB-185 (HUMO 36551, HUAP 75544) & $\mathrm{H}$ & $\mathrm{Na}$ \\
\hline Setaria grisebachii E. Fourn. & GMHB-286 (HUMO 36776, HUAP 75616) & $\mathrm{H}$ & $\mathrm{Na}$ \\
\hline Setaria liebmannii E. Fourn. & GMHB-282A (HUAP 75613), GMHB-382A (HUMO 367795) & $\mathrm{H}$ & $\mathrm{Na}$ \\
\hline Setaria macrostachya Kunth & AFM-371 (HUMO 36711) & $\mathrm{H}$ & $\mathrm{Na}$ \\
\hline Sporobolus atrovirens (Kunth) Kunth * & $\begin{array}{l}\text { GMHB-144 (HUMO 36989), GMHB-341 (HUMO } 36500 \\
\text { HUAP 75649), MJS-4 (HUMO 37619), RCT-3031 (HUMO } \\
\text { 36610) }\end{array}$ & $\mathrm{H}$ & $\mathrm{Na}$ \\
\hline
\end{tabular}


Apéndice: Continuación.

\begin{tabular}{|c|c|c|c|}
\hline Familias/especies & Ejemplares examinados & $\begin{array}{l}\text { Formas de } \\
\text { crecimiento }\end{array}$ & $\begin{array}{r}\text { Exótica/ } \\
\text { Nativa }\end{array}$ \\
\hline Sporobolus tenuissimus (Mart. ex Schrank) Kuntze $\bullet$ & GHMB-241 (HUMO 36645) & $\mathrm{H}$ & $\mathrm{Na}$ \\
\hline Tetrapogon chlorideus (J. Presl) P.M. Peterson & RCT-sn (HUMO 37658) & $\mathrm{H}$ & $\mathrm{Na}$ \\
\hline Trachypogon spicatus (L. f.) Kuntze & YMM-285 (HUMO 37627) & $\mathrm{H}$ & $\mathrm{Na}$ \\
\hline Tripsacum dactyloides (L.) L. & $\begin{array}{l}\text { GMHB-303B (HUMO 36474, HUAP 75626), RCT-3010 } \\
\text { (HUMO 36740, HUAP 75681), YMM-303 (HUMO 36899) }\end{array}$ & $\mathrm{H}$ & $\mathrm{Na}$ \\
\hline Tripsacum latifolium Hitchc. & AFM-374 (HUMO 36703) & $\mathrm{H}$ & $\mathrm{Na}$ \\
\hline \multicolumn{4}{|l|}{ MAGNOLIIDES } \\
\hline \multicolumn{4}{|l|}{ Piperaceae } \\
\hline Peperomia umbilicata Ruiz \& Pav. & GMHB-127A (HUMO 37071) & $\mathrm{H}$ & $\mathrm{Na}$ \\
\hline Peperomia schizandra Trel. * & GMHB-141 (HUMO 36421), MJS-24 (HUMO 37638) & $\mathrm{H}$ & $\mathrm{Na}$ \\
\hline \multicolumn{4}{|l|}{ EUDICOTILEDÓNEAS } \\
\hline \multicolumn{4}{|l|}{ Acanthaceae } \\
\hline Anisacanthus tulensis Greenm. * & $\begin{array}{l}\text { AFM-364 (HUMO 36602), GMHB-59 (HUMO 36462, HUAP } \\
\text { 75453) }\end{array}$ & $\mathrm{H}$ & $\mathrm{Na}$ \\
\hline Barleria oenotheroides Dum. Cours. & RCT-3036 (HUMO 36576, HUAP 75703) & $\operatorname{Ar}$ & $\mathrm{Na}$ \\
\hline $\begin{array}{l}\text { Carlowrightia neesiana (Schauer ex Nees) T.F. } \\
\text { Daniel* }\end{array}$ & $\begin{array}{l}\text { RCT-3000 (HUMO 36741, HUAP 75671), RCT-3066 (HUMO } \\
\text { 36967, HUAP 75724) }\end{array}$ & $\mathrm{H}$ & $\mathrm{Na}$ \\
\hline Carlowrightia pectinata Brandegee* & AFM-342 (HUMO 37651) & $\mathrm{H}$ & $\mathrm{Na}$ \\
\hline Dicliptera peduncularis Nees* & $\begin{array}{l}\text { AFM-317 (HUMO 36594), RCT-3004 (HUMO 36743, HUAP } \\
\text { 75675), RCT-3062 (HUMO 36972, HUAP 75722) }\end{array}$ & $\mathrm{H}$ & $\mathrm{Na}$ \\
\hline Elytraria imbricata (Vahl) Pers. & $\begin{array}{l}\text { GMHB-54 (HUMO 36432, HUAP 75434), GMHB-116A } \\
\text { (HUMO 37002), GMHB-349 (HUAP 75655), RCT-3007 } \\
\text { (HUAP 75678), RCT-3056 (HUAP 75717), RCT-3082 } \\
\text { (HUMO 36963, HUAP 75734) }\end{array}$ & $\mathrm{H}$ & $\mathrm{Na}$ \\
\hline Henrya insularis Nees & $\begin{array}{l}\text { GMHB-6 (HUMO 36443, HUAP 75448), RCT-3105 (HUMO } \\
\text { 36598, HUAP 75742) }\end{array}$ & $\operatorname{Ar}$ & $\mathrm{Na}$ \\
\hline Justicia caudata A. Gray & $\begin{array}{l}\text { GMHB-270 (HUMO 36770, HUAP 75604), GMHB-344 } \\
\text { (HUMO 36503, HUAP 75652) }\end{array}$ & $\operatorname{Ar}$ & $\mathrm{Na}$ \\
\hline Justicia hilsenbeckii T.F. Daniel* & $\begin{array}{l}\text { AFM-316 (HUMO 36959, HUAP 75819), RCT-3106 (HUAP } \\
\text { 75743) }\end{array}$ & $\mathrm{H}$ & $\mathrm{Na}$ \\
\hline Justicia salviiflora Kunth* & AFM-326 (HUMO 36725, HUAP 75826) & $\mathrm{H}$ & $\mathrm{Na}$ \\
\hline Ruellia nudiflora (Engelm. \& A. Gray) Urb. & GMHB-168 (HUMO 36672, HUAP 75531) & $\mathrm{H}$ & $\mathrm{Na}$ \\
\hline Tetramerium nervosum Nees & $\begin{array}{l}\text { AFM-330 (HUMO 36596), GMHB-356 (HUMO 36823, } \\
\text { HUAP } 75662 \text { ), RCT-3005 (HUMO 36745, HUAP 75676), } \\
\text { RCT-3060 (HUMO 36957), YMM-307A (HUMO 36836) }\end{array}$ & $\mathrm{H}$ & $\mathrm{Na}$ \\
\hline Tetramerium ochoterenae (Miranda) T.F. Daniel** & $\begin{array}{l}\text { AFM-369 (HUMO 37628), AFM-392 (HUMO 37644), } \\
\text { GMHB-2 (HUMO 36460, HUAP 75451) }\end{array}$ & $\operatorname{Ar}$ & $\mathrm{Na}$ \\
\hline \multicolumn{4}{|l|}{ Amaranthaceae } \\
\hline Amaranthus powellii S. Watson & YMM-339 (HUMO 36878) & $\mathrm{H}$ & $\mathrm{Na}$ \\
\hline $\begin{array}{l}\text { Chenopodiastrum murale (L.) S. Fuentes-B., Uotila } \\
\text { \& Borsch }\end{array}$ & GMHB-60B (HUMO 36388, HUAP 75467) & $\mathrm{H}$ & Ex \\
\hline Dysphania ambrosioides (L.) Mosyakin \& Clemants & GMHB-70 (HUMO 36937) & $\mathrm{H}$ & $\mathrm{Na}$ \\
\hline
\end{tabular}


Apéndice: Continuación.

\begin{tabular}{|c|c|c|c|}
\hline Familias/especies & Ejemplares examinados & $\begin{array}{l}\text { Formas de } \\
\text { crecimiento }\end{array}$ & $\begin{array}{r}\text { Exótica/ } \\
\text { Nativa }\end{array}$ \\
\hline Gomphrena serrata $\mathrm{L}$. & $\begin{array}{l}\text { GMHB-219 (HUMO 36695, HUAP 75567), GMHB-310 } \\
\text { (HUMO 36479), GMHB-321 (HUMO 36506, HUAP } \\
\text { 75637), RCT-3014 (HUMO 36979) }\end{array}$ & $\mathrm{H}$ & $\mathrm{Na}$ \\
\hline Iresine calea (Ibáñez) Standl. & $\begin{array}{l}\text { AFM-377 (HUMO 36712, HUAP 75856), RCT-3069 (HUMO } \\
\text { 36968, HUAP 75726) }\end{array}$ & $\mathrm{H}$ & $\mathrm{Na}$ \\
\hline Iresine diffusa Humb. \& Bonpl. ex Willd. & $\begin{array}{l}\text { AFM-370 (HUMO 36606), GMHB-9 (HUAP 75464), GMHB- } \\
21 \text { (HUMO 37662) }\end{array}$ & $\mathrm{H}$ & $\mathrm{Na}$ \\
\hline Iresine interrupta Benth. & AFM-327 (HUMO 36605, HUAP 75827) & $\mathrm{Ar}$ & $\mathrm{Na}$ \\
\hline \multicolumn{4}{|l|}{ Anacardiaceae } \\
\hline $\begin{array}{l}\text { Amphipterygium adstringens (Schltdl.) Standl. } \\
\text { subsp. adstringens } *\end{array}$ & $\begin{array}{l}\text { GMHB-131 (HUMO 36998, HUAP 75511), GMHB-107 } \\
\text { (HUMO 36990, HUAP 75498) }\end{array}$ & A & $\mathrm{Na}$ \\
\hline Spondias purpurea L. & $\begin{array}{l}\text { GMHB-52 (HUMO 36541, HUAP 75436), RCT-3068 } \\
\text { (HUMO 36976), YMM-293 (HUMO 36898) }\end{array}$ & A & $\mathrm{Na}$ \\
\hline \multicolumn{4}{|l|}{ Annonaceae } \\
\hline Annona reticulata $\mathrm{L}$. & RCT-3085 (HUMO 36953, HUAP 75737) & A & $\mathrm{Na}$ \\
\hline \multicolumn{4}{|l|}{ Apiaceae } \\
\hline Prionosciadium nelsonii J.M. Coult. \& Rose** & GMHB-189 (HUMO 36389, HUAP 75547) & $\mathrm{H}$ & $\mathrm{Na}$ \\
\hline \multicolumn{4}{|l|}{ Apocynaceae } \\
\hline Asclepias curassavica $\mathrm{L}$. & GMHB-73 (HUMO 36672) & $\mathrm{H}$ & $\mathrm{Na}$ \\
\hline Asclepias oenotheroides Schldl. \& Cham. & AFM-415 (HUMO 37635) & $\mathrm{H}$ & $\mathrm{Na}$ \\
\hline Cynanchum foetidum (Cav.) Kunth $*$ & AFM-391 (HUMO 36719) & $\mathrm{T}$ & $\mathrm{Na}$ \\
\hline Funastrum clausum (Jacq.) Schltr. & MJS-22 (HUMO 37655) & $\mathrm{T}$ & $\mathrm{Na}$ \\
\hline Funastrum pannosum Schltr.** & AFM-296 (MEXU 1482724) & $\mathrm{T}$ & $\mathrm{Na}$ \\
\hline Gonolobus pectinatus Brandegee* & $\begin{array}{l}\text { GMHB-229 (HUMO 366675, HUAP 75576), RCT-3082 } \\
\text { (HUMO 36981) }\end{array}$ & $\mathrm{T}$ & $\mathrm{Na}$ \\
\hline $\begin{array}{l}\text { Laubertia contorta (M. Martens \& Galeotti) } \\
\text { Woodson* }\end{array}$ & $\begin{array}{l}\text { GMHB-117 (HUMO 37015, HUAP 75501), YMM-284 } \\
\text { (HUMO 36858) }\end{array}$ & $\mathrm{T}$ & $\mathrm{Na}$ \\
\hline Macroscepis pleistantha Donn. Sm. & AFM-313 (MEXU 1494830) & $\mathrm{T}$ & $\mathrm{Na}$ \\
\hline $\begin{array}{l}\text { Mandevilla holosericea (Sessé \& Moc.) J.K. } \\
\text { Williams* }\end{array}$ & MIMV-477 (HUMO 37647) & $\mathrm{T}$ & $\mathrm{Na}$ \\
\hline Marsdenia zimapanica Hemsl. * & GMHB-55 (HUMO 36510, HUAP 75435) & $\mathrm{T}$ & $\mathrm{Na}$ \\
\hline Matelea crenata (Vail) Woodson* & $\begin{array}{l}\text { GMHB-288 (HUMO 36774, HUAP 75617), MJS-11 (HUMO } \\
\text { 37641) }\end{array}$ & $\mathrm{T}$ & $\mathrm{Na}$ \\
\hline Matelea trachyantha (Greenm.) W.D. Stevens & $\begin{array}{l}\text { GMHB-358 (HUMO 36862, HUAP 75663), LORM } 78 \\
\text { (MEXU 1482717) }\end{array}$ & $\mathrm{Ar}$ & $\mathrm{Na}$ \\
\hline Plumeria rubra $L$. & $\begin{array}{l}\text { AFM-394 (HUMO 36757, HUAP 75866), GMHB-4 (HUAP } \\
\text { 75449) }\end{array}$ & $A$ & $\mathrm{Na}$ \\
\hline Polystemma guatemalense (Schltr.) W.D. Stevens & $\begin{array}{l}\text { AFM-314 (HUMO 36604), GMHB-287 (HUMO 36493), } \\
\text { GMHB-292 (HUMO 36570, HUAP 75618), RCT-3086 } \\
\text { (HUMO 36980) }\end{array}$ & $T$ & $\mathrm{Na}$ \\
\hline \multicolumn{4}{|l|}{ Araliaceae } \\
\hline Aralia humilis Cav. & GMHB-259 (HUAP 75596), MJS-14 (HUMO 37643) & $\mathrm{Ar}$ & $\mathrm{Na}$ \\
\hline
\end{tabular}


Apéndice: Continuación.

\begin{tabular}{|c|c|c|c|}
\hline Familias/especies & Ejemplares examinados & $\begin{array}{l}\text { Formas de } \\
\text { crecimiento }\end{array}$ & $\begin{array}{r}\text { Exótica/ } \\
\text { Nativa }\end{array}$ \\
\hline \multicolumn{4}{|l|}{ Asteraceae } \\
\hline \multirow[t]{2}{*}{ Acmella radicans (Jacq.) R.K. Jansen } & RCT-3006 (HUMO 36744, HUAP 75677) & \multirow[t]{2}{*}{$\mathrm{H}$} & \multirow[t]{2}{*}{$\mathrm{Na}$} \\
\hline & YMM-332 (HUMO 36935, HUAP 75784), YMM-333 & & \\
\hline Ageratum corymbosum Zuccagni & $\begin{array}{l}\text { (HUMO 36943), YMM-334 (HUAP 75785), YMM-335 } \\
\text { (HUMO 36938) }\end{array}$ & $\mathrm{H}$ & $\mathrm{Na}$ \\
\hline Alomia alata Hemsl. $*$ & $\begin{array}{l}\text { AFM-315 (HUMO 36575), GMHB-32 (HUMO 36505), RCT- } \\
3064 \text { (HUMO 36969, HUAP 75723) }\end{array}$ & $\mathrm{H}$ & $\mathrm{Na}$ \\
\hline Baccharis salicifolia (Ruiz \& Pav.) Pers. & MJS-s.n. (HUMO 37648) & $\mathrm{Ar}$ & $\mathrm{Na}$ \\
\hline Bidens odorata Cav. & YMM-278 (HUMO 37605) & $\mathrm{H}$ & $\mathrm{Na}$ \\
\hline Bidens sp. & GMHB-360 (HUMO 36886, HUAP 75665) & $\mathrm{H}$ & - \\
\hline Brickellia diffusa (Vahl) A. Gray & RCT-3038 (HUMO 36582, HUAP 75704) & $\mathrm{H}$ & $\mathrm{Na}$ \\
\hline Brickellia glandulosa (La Llave) McVaugh & AFM-385 (HUMO 36755, HUAP 758620) & $\mathrm{H}$ & $\mathrm{Na}$ \\
\hline Chromolaena odorata (L.) R.M. King \& H. Rob. & RCT-3074 (HUMO 36956, HUAP 75731) & $\operatorname{Ar}$ & $\mathrm{Na}$ \\
\hline Dahlia coccinea Cav. & GMHB-222 (HUMO 36964), GMHB-345 (HUMO 36803) & $\mathrm{H}$ & $\mathrm{Na}$ \\
\hline Delilia biflora (L.) Kuntze & $\begin{array}{l}\text { GMHB-341 (HUAP 75650), GMHB-342 (HUMO 36497), } \\
\text { YMM-362 (HUMO 36948, HUAP 75750) }\end{array}$ & $\mathrm{H}$ & $\mathrm{Na}$ \\
\hline Eclipta prostrata (L.) L. & AFM-357 (HUAP 75848) & $\mathrm{H}$ & $\mathrm{Na}$ \\
\hline Fleischmannia arguta (Kunth) B.L. Rob. & GMHB-65 (HUMO 36395, HUAP 75469) & $\mathrm{H}$ & $\mathrm{Na}$ \\
\hline Florestina pedata (Cav.) Cass. & $\begin{array}{l}\text { GMHB-232 (HUMO 36693, HUAP 75578), RCT-3101 } \\
\text { (HUMO 36966), YMM-363 (HUMO 36842, HUAP 75751) }\end{array}$ & $\mathrm{H}$ & $\mathrm{Na}$ \\
\hline Galeana pratensis (Kunth) Rydb. & GMHB-180 (HUMO 36660, HUAP 75539) & $\mathrm{H}$ & $\mathrm{Na}$ \\
\hline Galinsoga parviflora Cav. & YMM-311 (HUMO 36853) & $\mathrm{H}$ & $\mathrm{Na}$ \\
\hline Lasianthaea crocea (A. Gray) K.M. Becker* & $\begin{array}{l}\text { GMHB-347 (HUMO 37646, HUAP 75654), RCT-3037 } \\
\text { (HUMO 36786, HUAP 75702) }\end{array}$ & $\mathrm{Ar}$ & $\mathrm{Na}$ \\
\hline Laphangium luteoalbum (L.) Tzvelev & AFM-356 (HUAP 75847) & $\mathrm{H}$ & Ex \\
\hline Melampodium linearilobum DC. & RCT-3020A (HUMO 36729, HUAP 75689) & $\mathrm{H}$ & $\mathrm{Na}$ \\
\hline Milleria quinqueflora $\mathrm{L}$. & YMM-360 (HUMO 36873, HUAP 75752) & $\mathrm{H}$ & $\mathrm{Na}$ \\
\hline Montanoa grandiflora DC. * & GMHB-265 (HUAP 75600), YMM-302 (HUMO 36900) & $\operatorname{Ar}$ & $\mathrm{Na}$ \\
\hline Otopappus epaleaceus Hemsl. ** & $\begin{array}{l}\text { GMHB-314 (HUMO 36478, HUAP 75630), GMHB-350 } \\
\text { (HUMO 36827, HUAP 75657) }\end{array}$ & $\mathrm{Ar}$ & $\mathrm{Na}$ \\
\hline Pectis haenkeana (DC.) Sch. Bip. * & $\begin{array}{l}\text { MJS-12 (HUMO 37640), YMM-295 (HUMO 36907, HUAP } \\
\text { 75811) }\end{array}$ & $\mathrm{H}$ & $\mathrm{Na}$ \\
\hline Pectis prostrata Cav. & $\begin{array}{l}\text { AFM-361 (HUMO 36616), GMHB-304 (HUMO 36483), } \\
\text { GMHB-328 (HUMO 36430), YMM-338 (HUMO 36826) }\end{array}$ & $\mathrm{H}$ & $\mathrm{Na}$ \\
\hline Pectis uniaristata DC. var. uniaristata & $\begin{array}{l}\text { GMHB-308 (HUMO 36480), GMHB-322 (HUMO 36450, } \\
\text { HUAP 75638) }\end{array}$ & $\mathrm{H}$ & $\mathrm{Na}$ \\
\hline $\begin{array}{l}\text { Perymenium mendezii DC. var. verbesinoides (DC.) } \\
\text { J.J. Fay* }\end{array}$ & GMHB-209 (HUMO 36680, HUAP 75558) & $\mathrm{Ar}$ & $\mathrm{Na}$ \\
\hline Pittocaulon praecox (Cav.) H. Rob. \& Brettell * & $\begin{array}{l}\text { AFM-396 (HUMO 36722), GMHB-28 (HUMO 36463, HUAP } \\
\text { 75455), YMM-317 (HUMO 36890, HUAP 75774) }\end{array}$ & $\mathrm{Ar}$ & $\mathrm{Na}$ \\
\hline $\begin{array}{l}\text { Pittocaulon velatum (Greenm.) H. Rob. \& Brettell } \\
\text { var. velatum }\end{array}$ & AFM-372 (HUMO 36720) & $\mathrm{Ar}$ & $\mathrm{Na}$ \\
\hline
\end{tabular}


Apéndice: Continuación.

\begin{tabular}{|c|c|c|c|}
\hline Familias/especies & Ejemplares examinados & $\begin{array}{l}\text { Formas de } \\
\text { crecimiento }\end{array}$ & $\begin{array}{r}\text { Exótica/ } \\
\text { Nativa }\end{array}$ \\
\hline $\begin{array}{l}\text { Porophyllum ruderale (Jacq.) Cass. subsp. } \\
\text { macrocephalum (DC.) R.R. Johnson }\end{array}$ & $\begin{array}{l}\text { GMHB-1 (HUMO 36399, HUAP 75452), YMM-300 (HUMO } \\
\text { 36902, HUAP 75814) }\end{array}$ & $\mathrm{H}$ & $\mathrm{Na}$ \\
\hline $\begin{array}{l}\text { Pseudelephantopus spicatus (B. Juss. ex Aubl.) C.F. } \\
\text { Baker }\end{array}$ & AFM-352 (HUMO 36619, HUAP 75845) & $\mathrm{H}$ & $\mathrm{Na}$ \\
\hline Sanvitalia procumbens Lam. & GMHB-316 (HUMO 36465, HUAP 75632) & $\mathrm{H}$ & $\mathrm{Na}$ \\
\hline Schkuhria pinnata (Lam.) Kuntze ex Thell. & YMM-331 (HUMO 36911, HUAP 75782) & $\mathrm{H}$ & $\mathrm{Na}$ \\
\hline Simsia sanguinea A. Gray & $\begin{array}{l}\text { GMHB-217 (HUMO 36697, HUAP 75566), YMM-270 } \\
\text { (HUMO 36908, HUAP 75797), YMM-335 (HUMO 36938, } \\
\text { HUAP 75786) }\end{array}$ & $\mathrm{H}$ & $\mathrm{Na}$ \\
\hline Stevia ovata Willd. & RCT-3012 (HUMO 36748, HUAP 75683) & $\mathrm{H}$ & $\mathrm{Na}$ \\
\hline Stevia trifida Lag. * & AFM-358 (HUAP 75849, HUMO 36588) & $\mathrm{H}$ & $\mathrm{Na}$ \\
\hline Symphyotrichum subulatum (Michx.) G.L. Nesom & GMHB-74 (HUMO 36370, HUAP 75474) & $\mathrm{H}$ & $\mathrm{Na}$ \\
\hline Tagetes lunulata Ortega* & $\begin{array}{l}\text { GMHB-300 (HUMO 36473), GMHB-337 (HUMO 36509, } \\
\text { HUAP 75646), YMM-346 (HUMO 36857) }\end{array}$ & $\mathrm{H}$ & $\mathrm{Na}$ \\
\hline Tithonia tubaeformis (Jacq.) Cass. & AFM-341 (HUMO 36618), RCT-3103 (HUMO 36951) & $\mathrm{H}$ & $\mathrm{Na}$ \\
\hline Tridax coronopifolia (Kunth) Hemsl.** & $\begin{array}{l}\text { GMHB-143 (HUMO 37003), GMHB-233A (HUMO 36687), } \\
\text { YMM-343 (HUAP 75789) }\end{array}$ & $\mathrm{H}$ & $\mathrm{Na}$ \\
\hline Tridax mexicana A.M. Powell* & GMHB-126 (HUAP 75507) & $\mathrm{H}$ & $\mathrm{Na}$ \\
\hline Tridax procumbens L. & GMHB-233B (HUMO 36663, HUAP 75579) & $\mathrm{H}$ & $\mathrm{Na}$ \\
\hline Verbesina crocata (Cav.) Less. & RCT-3040 (HUMO 36785, HUAP 75706) & $\operatorname{Ar}$ & $\mathrm{Na}$ \\
\hline Verbesina oncophora B.L. Rob. \& Seaton* & GMHB-315 (HUMO 36501, HUAP 75631) & $\operatorname{Ar}$ & $\mathrm{Na}$ \\
\hline Vernonia liatroides DC. $*$ & $\begin{array}{l}\text { AFM-347 (HUMO 36611, HUAP 75840), RCT-3017B (HUAP } \\
\text { 75687) }\end{array}$ & $\operatorname{Ar}$ & $\mathrm{Na}$ \\
\hline Zinnia americana (Mill.) Olorode \& A.M. Torres & $\begin{array}{l}\text { GMHB-354 (HUMO 36865, HUAP 75661), YMM-281 } \\
\text { (HUAP 75804) }\end{array}$ & $\mathrm{H}$ & $\mathrm{Na}$ \\
\hline Zinnia peruviana (L.) L. & $\begin{array}{l}\text { GMHB-194 (HUMO 36572, HUAP 75550), GMHB-254 } \\
\text { (HUMO 36778, HUAP 75595) }\end{array}$ & $\mathrm{H}$ & $\mathrm{Na}$ \\
\hline \multicolumn{4}{|l|}{ Begoniaceae } \\
\hline Begonia gracilis Kunth & $\begin{array}{l}\text { GMHB-103B (HUMO 37010, HUAP 75495), GMHB-104 } \\
\text { (HUMO 37075, HUAP 75496) }\end{array}$ & $\mathrm{H}$ & $\mathrm{Na}$ \\
\hline Bignoniaceae & & & $\mathrm{Na}$ \\
\hline Amphilophium crucigerum (L.) L.G. Lohmann & $\begin{array}{l}\text { GMHB-39 (HUMO 36428), GMHB-103A (HUMO 36999, } \\
\text { HUAP 75494) }\end{array}$ & $\mathrm{T}$ & $\mathrm{Na}$ \\
\hline Crescentia alata Kunth & GMHB-165 (HUMO 36673) & A & $\mathrm{Na}$ \\
\hline $\begin{array}{l}\text { Tecoma stans (L.) Juss. ex Kunth var. angustata } \\
\text { Rehder }\end{array}$ & GMHB-177 (HUMO 36667, HUAP 75537) & $A$ & $\mathrm{Na}$ \\
\hline \multicolumn{4}{|l|}{ Brassicaceae } \\
\hline Lepidium virginicum $\mathrm{L}$. & AFM-338 (HUMO 36581), RCT-3109 (HUMO 37613) & $\mathrm{H}$ & $\mathrm{Na}$ \\
\hline \multicolumn{4}{|l|}{ Burseraceae } \\
\hline Bursera bicolor (Willd. ex Schltdl.) Engl. & GMHB-51 (HUMO 36439, HUAP 75437) & A & $\mathrm{Na}$ \\
\hline Bursera copallifera (DC.) Bullock* & GMHB-45 (HUMO 36427, HUAP 75442) & A & $\mathrm{Na}$ \\
\hline Bursera fagaroides (Kunth) Engl. & AFM-378 (HUMO 36762, HUAP 75857) & A & $\mathrm{Na}$ \\
\hline Bursera schlechtendalii Engl. & GMHB-160 (HUMO 36656, HUAP 75527) & A & $\mathrm{Na}$ \\
\hline
\end{tabular}


Apéndice: Continuación.

\section{Familias/especies}

Ejemplares examinados

Formas de

crecimiento
Exótica/ Nativa

\section{Cactaceae}

Coryphantha elephantidens (Lem.) Lem. $* \diamond$

AFM-sna (HUMO 37597)

AFM-snb (HUMO 37599)

Isolatocereus dumortieri (Scheidw.) Backeb. *

AFM-412 (HUMO 36855)

AFM-snc (HUMO 37598)

GMHB-27 (HUMO 36425)

Myrtillocactus geometrizans (Mart. ex Pfeiff.)

Console*

Opuntia decumbens Salm-Dyck

AFM-snd (HUMO 37600)

Opuntia fuliginosa Griffiths

Opuntia pubescens H.L. Wendl. ex Pfeiff.

Pereskiopsis diguetii (F.A.C. Weber) Britton \& Rose*

Pereskiopsis kellermanii Rose*

Stenocereus stellatus (Pfeiff.) Riccob. *

\section{Campanulaceae}

Calcaratolobelia cordifolia (Hook. \& Arn.) Wilbur

Diastatea micrantha (Kunth) McVaugh

Caryophyllaceae

Drymaria gracilis Schltdl. \& Cham.

Drymaria villosa Schltdl. \& Cham.

\section{Cleomaceae}

Cleome guianensis Aubl.

\section{Convolvulaceae}

Evolvulus alsinoides (L.) L.

Ipomoea bracteata Cav. *

Ipomoea conzattii Greenm. *

Ipomoea costellata Torr.

Ipomoea murucoides Roem. \& Schult.

Ipomoea parasitica (Kunth) G. Don.

Ipomoea pauciflora M. Martens \& Galeotti

Ipomoea sp.

\section{Cordiaceae}

Varronia curassavica Jacq.

Varronia globosa Jacq.

AFM-sne (HUMO 37602), GMHB-167 (HUMO 36657, HUAP 75530)

AFM-snf (HUMO 37601)

MJS-20 (HUMO 37615)

RCT-3070 (HUAP 75727)

GMHB-202 (HUMO 36753)

AFM-353 (HUMO 36580, HUAP 75846), RCT-3026 (HUMO 36738, HUAP 75694)

YMM-361 (HUMO 36861, HUAP 75749)

AFM-337 (HUMO 36724, HUAP 75834)

GMHB-313 (HUMO 36477)

GMHB-183 (HUMO 36563, HUAP 7542), GMHB-340

(HUMO 36490, HUAP 75648)

GMHB-99 (HUMO 36440), GMHB-147 (HUMO 36884),

RCT-3073 (HUAP 75730), YMM-342 (HUMO 36933)

AFM-363 (HUMO 36614)

AFM-390 (HUAP 75864), GMHB-3 (HUMO 36445, HUAP

75450), GMHB-359 (HUMO 36829, HUAP 75664)

YMM-297 (HUMO 36905, HUAP 75813)

GMHB-324 (HUMO 36338, HUAP 75639), RCT-3050

(HUMO 36710), YMM-272 (HUMO 36915, HUAP 75799)

YMM-271 (HUMO 36914, HUAP 75798)

RCT-3034 (HUMO 36807), RCT-3049 (HUMO 36726, HUAP 75713)

YMM-285 (HUMO 36921), GMHB-323 (HUMO 37620)

GMHB-190 (HUMO 36554, HUAP 75548)

GMHB-156 (HUAP 75524), GMHB-212 (HUMO 36628, HUAP 75560)
G

$\mathrm{Na}$

$\mathrm{Na}$

$\mathrm{Na}$

$\mathrm{Na}$

$\mathrm{Na}$

Can $\quad \mathrm{Na}$

$\mathrm{Ar} \quad \mathrm{Na}$

Ar $\quad \mathrm{Na}$

$\mathrm{Ar} \quad \mathrm{Na}$

$\mathrm{Ar} \quad \mathrm{Na}$

Can $\quad \mathrm{Na}$

Can $\quad \mathrm{Na}$

$\mathrm{H} \quad \mathrm{Na}$

$\mathrm{H}$

$\mathrm{Na}$

$\mathrm{H}$

$\mathrm{Na}$

$\mathrm{H}$

$\mathrm{Na}$

$\mathrm{H}$

$\mathrm{H}$

$\mathrm{T}$

$\mathrm{T}$

$\mathrm{T}$

A

$\mathrm{T}$

E

$\mathrm{Ar}$

$\mathrm{Na}$

$\operatorname{Ar}$

$\mathrm{Na}$
$\mathrm{Ar} \quad \mathrm{Na}$ 
Apéndice: Continuación.

\begin{tabular}{|c|c|c|c|}
\hline Familias/especies & Ejemplares examinados & $\begin{array}{l}\text { Formas de } \\
\text { crecimiento }\end{array}$ & $\begin{array}{r}\text { Exótica/ } \\
\text { Nativa }\end{array}$ \\
\hline Varronia guanacastensis (Standl.) J.S. Mill. • & $\begin{array}{l}\text { GMHB-100 (HUMO 36983, HUAP 75491), GMHB-156 } \\
\text { (HUMO 36640) }\end{array}$ & $\mathrm{H}$ & $\mathrm{Na}$ \\
\hline \multicolumn{4}{|l|}{ Crassulaceae } \\
\hline Sedum sp. & YMM-280 (HUMO 36916) & $\mathrm{H}$ & - \\
\hline Thompsonella minutiflora (Rose) Britton \& Rose* & GMHB-218 (HUMO 36475, HUAP 75564) & $\mathrm{H}$ & $\mathrm{Na}$ \\
\hline \multicolumn{4}{|l|}{ Cucurbitaceae } \\
\hline Echinopepon racemosus (Steud.) C. Jeffrey & YMM-358 (HUMO 36889) & $\mathrm{T}$ & $\mathrm{Na}$ \\
\hline Melothria pendula $\mathrm{L}$. & $\begin{array}{l}\text { GMHB-275A (HUMO 36766), GMHB-282B (HUMO 36794, } \\
\text { HUAP 75614), MJS-26 (HUMO 37636), YMM-327 } \\
\text { (HUMO 36937, HUAP 75780) }\end{array}$ & $\mathrm{T}$ & $\mathrm{Na}$ \\
\hline \multicolumn{4}{|l|}{ Ebenaceae } \\
\hline Diospyros salicifolia Humb. \& Bonpl. ex Willd. & $\begin{array}{l}\text { AFM-393 (HUMO 36754, HUAP 75865), RCT-3048 (HUMO } \\
\text { 36788, HUAP 75712) }\end{array}$ & A & $\mathrm{Na}$ \\
\hline \multicolumn{4}{|l|}{ Euphorbiaceae } \\
\hline Acalypha sp. & AFM-373 (HUMO 36761) & $\mathrm{H}$ & - \\
\hline Cnidoscolus multilobus (Pax) I.M. Johnst. & MIMV-479 (HUMO 36700, HUAP 75871) & A & $\mathrm{Na}$ \\
\hline $\begin{array}{l}\text { Cnidoscolus rostratus Lundell subsp. glabratus } \\
\text { Breckon* }\end{array}$ & GMHB 94B (HUMO 36461, HUAP 75486) & A & $\mathrm{Na}$ \\
\hline Cnidoscolus sp. & MJS-1 (HUMO 37630) & A & - \\
\hline Croton ciliatoglandulifer Ortega & GMHB-149 (HUMO 36853) & $\operatorname{Ar}$ & $\mathrm{Na}$ \\
\hline Croton morifolius Willd. & GMHB-111 (HUAP 75499) & $\operatorname{Ar}$ & $\mathrm{Na}$ \\
\hline Euphorbia breedlovei V. W. Steinm. \& P. Carrillo* & YMM-377 (HUMO 36939, HUAP 75787) & $\mathrm{H}$ & $\mathrm{Na}$ \\
\hline Euphorbia graminea Jacq. & RCT-3035 (HUMO 36715) & $\mathrm{H}$ & $\mathrm{Na}$ \\
\hline Euphorbia hirta L. & $\begin{array}{l}\text { AFM-332 (HUMO 36624), GMHB-66 (HUMO 36996, HUAP } \\
\text { 75470), GMHB-105 (HUMO 36996), RCT-3065 (HUMO } \\
\text { 36964) }\end{array}$ & $\mathrm{H}$ & $\mathrm{Na}$ \\
\hline Euphorbia indivisa (Engelm.) Tidestr. & YMM359 (HUMO 36989) & $\mathrm{H}$ & $\mathrm{Na}$ \\
\hline $\begin{array}{l}\text { Euphorbia schiedeana (Klotzsch \& Garcke) Mayfield } \\
\text { ex C. Nelson }\end{array}$ & GMHB-243 (HUMO 36647, HUAP 75585) & $\mathrm{H}$ & $\mathrm{Na}$ \\
\hline Euphorbia schlechtendalii Boiss. & GMHB-19 (HUMO 36809) & $\operatorname{Ar}$ & $\mathrm{Na}$ \\
\hline Jatropha dioica Sessé ex Cerv. & $\begin{array}{l}\text { GMHB-44 (HUMO 36504, HUAP 75443), GMHB-47 } \\
\text { (HUMO 36441, HUAP 75440), GMHB-106 (HUMO } \\
\text { 37013, HUAP 75497) }\end{array}$ & $\operatorname{Ar}$ & $\mathrm{Na}$ \\
\hline Manihot angustiloba (Torr.) Müll. Arg. & GMHB-193 (HUAP 75549) & $\operatorname{Ar}$ & $\mathrm{Na}$ \\
\hline Sapium macrocarpum Müll. Arg. $\diamond$ & GMHB-146 (HUMO 36885) & A & $\mathrm{Na}$ \\
\hline \multicolumn{4}{|l|}{ Fabaceae } \\
\hline $\begin{array}{l}\text { Acaciella painteri Britton \& Rose var. houghii } \\
\text { (Britton \& Rose) L. Rico* }\end{array}$ & $\begin{array}{l}\text { AFM-365 (HUMO 36698), GMHB-331A (HUMO 36467, } \\
\text { HUAP 75643) }\end{array}$ & $\operatorname{Ar}$ & $\mathrm{Na}$ \\
\hline Aeschynomene americana $\mathrm{L}$. & $\begin{array}{l}\text { GMHB-352 (HUMO 36833, HUAP 75659), RCT-3017A } \\
\text { (HUMO 36727), YMM-355 (HUMO 36824) }\end{array}$ & $\mathrm{H}$ & $\mathrm{Na}$ \\
\hline Brongniartia podalyrioides Kunth $*$ & $\begin{array}{l}\text { GMHB-226 (HUMO 36686, HUAP 75571), YMM-296 } \\
\text { (HUMO 36906, HUAP 75812) }\end{array}$ & $\operatorname{Ar}$ & $\mathrm{Na}$ \\
\hline
\end{tabular}


Apéndice: Continuación.

\begin{tabular}{|c|c|c|c|}
\hline Familias/especies & Ejemplares examinados & $\begin{array}{l}\text { Formas de } \\
\text { crecimiento }\end{array}$ & $\begin{array}{r}\text { Exótica/ } \\
\text { Nativa }\end{array}$ \\
\hline Calliandra eriophylla Benth. & $\begin{array}{l}\text { GMHB-43 (HUMO 36489), GMHB-46 (HUMO 36494, } \\
\text { HUAP 75441) }\end{array}$ & $\mathrm{Ar}$ & $\mathrm{Na}$ \\
\hline Calliandra houstoniana (Mill.) Standl. & GMHB-247 (HUMO 36663, HUAP 75589) & $\mathrm{Ar}$ & $\mathrm{Na}$ \\
\hline Canavalia villosa Benth. & AFM-318 (HUAP 75820) & $\mathrm{T}$ & $\mathrm{Na}$ \\
\hline Conzattia multiflora (B.L. Rob.) Standl. * & GMHB-57 (HUMO 36495) & A & $\mathrm{Na}$ \\
\hline Crotalaria cajanifolia Kunth & GMHB-86 (HUMO 36431, HUAP 75480) & $\mathrm{H}$ & $\mathrm{Na}$ \\
\hline Crotalaria mollicula Kunth & GMHB-339 (HUMO 36419), GMHB-373A (HUMO 36356) & $\mathrm{Ar}$ & $\mathrm{Na}$ \\
\hline Crotalaria pumila Ortega & GMHB-373B (HUMO 36361, HUAP 75670) & $\operatorname{Ar}$ & $\mathrm{Na}$ \\
\hline $\begin{array}{l}\text { Chamaecrista nictitans (L.) Moench var. mensalis } \\
\text { (Greenm.) H.S. Irwin \& Barneby }\end{array}$ & GMHB-215 (HUMO 36679), GMHB-245A (HUMO 37626) & $\operatorname{Ar}$ & $\mathrm{Na}$ \\
\hline $\begin{array}{l}\text { Chamaecrista nictitans var. jaliscensis (Greenm.) } \\
\text { H.S. Irwin \& Barneby }\end{array}$ & GMHB-352A (HUMO 36358, HUAP 75653) & $\mathrm{Ar}$ & $\mathrm{Na}$ \\
\hline Dalea foliolosa (Aiton) Barneby & GMHB-361 (HUMO 35821) & $\mathrm{H}$ & $\mathrm{Na}$ \\
\hline Dalea humilis G. Don & $\begin{array}{l}\text { GMHB-327 (HUMO 36484, HUAP 75641), RCT-3008 } \\
\text { (HUAP 75679), RCT-3100 (HUMO 36970, HUAP 75471), } \\
\text { YMM-283 (HUAP 75805) }\end{array}$ & $\mathrm{H}$ & $\mathrm{Na}$ \\
\hline Dalea leptostachya DC. * & GMHB-349A (HUMO 36403, HUAP 75656) & $\operatorname{Ar}$ & $\mathrm{Na}$ \\
\hline Desmanthus virgatus (L.) Willd. & GMHB-161 (HUMO 36655) & $\mathrm{H}$ & $\mathrm{Na}$ \\
\hline Desmodium procumbens (Mill.) Hitchc. & YMM-336 (HUMO 36944), GMHB-363 (HUMO 37623) & $\mathrm{H}$ & $\mathrm{Na}$ \\
\hline Desmodium sp. & $\begin{array}{l}\text { RCT-3019B (HUMO 36731, HUAP 75688), YMM-304 } \\
\text { (HUMO 36894, HUAP 75817) }\end{array}$ & $\mathrm{H}$ & - \\
\hline Diphysa suberosa S. Watson* & GMHB-163 (HUMO 36669, HUAP 75521) & $\operatorname{Ar}$ & $\mathrm{Na}$ \\
\hline Diphysa sp. & $\begin{array}{l}\text { GMHB-123 (HUMO 37014, HUAP 75505), GMHB-249 } \\
\text { (HUMO 36664, HUAP 75591) }\end{array}$ & $\mathrm{Ar}$ & - \\
\hline Eysenhardtia polystachya (Ortega) Sarg. & RCT-3053 (HUMO 36892, HUAP 75716) & A & $\mathrm{Na}$ \\
\hline Galactia viridiflora (Rose) Standl. * & YMM-309 (HUAP 75769), YMM-310 (HUMO 36480) & $\mathrm{E}$ & $\mathrm{Na}$ \\
\hline Leucaena macrophylla Benth. * & GMHB-53 (HUMO 36469, HUAP 75433) & A & $\mathrm{Na}$ \\
\hline Lonchocarpus eriophyllus Benth. * & GMHB-84 (HUMO 36442, HUAP 75478) & Ar & $\mathrm{Na}$ \\
\hline $\begin{array}{l}\text { Lysiloma acapulcense (Kunth) Benth. var. } \\
\text { acapulcense }\end{array}$ & YMM-315 (HUMO 36852, HUAP 75772) & A & $\mathrm{Na}$ \\
\hline Macroptilium atropurpureum (DC.) Urb. & $\begin{array}{l}\text { GMHB-237 (HUMO 36689, HUAP 75582), YMM-314 } \\
\text { (HUMO 36851, HUAP 75771) }\end{array}$ & $\mathrm{T}$ & $\mathrm{Na}$ \\
\hline Macroptilium gibbosifolium (Ortega) A. Delgado & GMHB-299A (HUMO 36764, HUAP 75623) & $\mathrm{T}$ & $\mathrm{Na}$ \\
\hline Macroptilium pedatum (Rose) Maréchal \& Baudet米 & GMHB-216 (HUMO 36682), GMHB-299B (HUMO 36777) & $\mathrm{T}$ & $\mathrm{Na}$ \\
\hline Marina scopa Barneby & RCT-3102 (HUMO 36962) & $\mathrm{H}$ & $\mathrm{Na}$ \\
\hline $\begin{array}{l}\text { Mariosousa salazarii (Briton \& Rose) Seigler \& } \\
\text { Ebinger }\end{array}$ & $\begin{array}{l}\text { AFM-400 (HUMO 36705, HUAP 75868), GMHB-13 (HUMO } \\
\text { 36418, HUAP 75461), RCT-3057 (HUMO 36984, HUAP } \\
\text { 75718) }\end{array}$ & A & $\mathrm{Na}$ \\
\hline Mimosa albida Humb. \& Bonpl. ex Willd. & $\begin{array}{l}\text { GMHB-267 (HUMO 36556, HUAP 75562), RCT-3022 } \\
\text { (HUMO 36751, HUAP 75690) }\end{array}$ & $\operatorname{Ar}$ & $\mathrm{Na}$ \\
\hline Mimosa lacerata Rose* & YMM-318 (HUMO 36839) & A & $\mathrm{Na}$ \\
\hline Mimosa polyantha Benth. & GMHB-89 (HUMO 36383, HUAP 75483) & $\mathrm{Ar}$ & $\mathrm{Na}$ \\
\hline
\end{tabular}


Apéndice: Continuación.

\begin{tabular}{|c|c|c|c|}
\hline Familias/especies & Ejemplares examinados & $\begin{array}{l}\text { Formas de } \\
\text { crecimiento }\end{array}$ & $\begin{array}{r}\text { Exótica/ } \\
\text { Nativa }\end{array}$ \\
\hline Nissolia fruticosa Jacq. & $\begin{array}{l}\text { GMHB-157 (HUMO 36650, HUAP 75525), GMHB-175 } \\
\text { (HUMO 36655, HUAP 75536), YMM-315 (HUMO 36852) }\end{array}$ & $\mathrm{T}$ & $\mathrm{Na}$ \\
\hline Pachyrhizus erosus (L.) Urb. & MJS-9 (HUMO 37629) & $\mathrm{T}$ & $\mathrm{Na}$ \\
\hline Phaseolus anisotrichos Schltdl. & YMM-357 (HUMO 36848, HUAP 75756) & $\mathrm{T}$ & $\mathrm{Na}$ \\
\hline Phaseolus microcarpus Mart. * & $\begin{array}{l}\text { GMHB-282C (HUMO 36881, HUAP 75612), YMM-340 } \\
\text { (HUMO 36820, HUAP 75788) }\end{array}$ & $\mathrm{T}$ & $\mathrm{Na}$ \\
\hline Pithecellobium dulce (Roxb.) Benth. & GMHB-25 (HUAP 75457) & A & $\mathrm{Na}$ \\
\hline $\begin{array}{l}\text { Rhynchosia precatoria (Humb. \& Bonpl. ex Willd.) } \\
\text { DC. }\end{array}$ & $\begin{array}{l}\text { AFM-367 (HUMO 36612, HUAP 75854), GMHB-5 (HUMO } \\
\text { 36434), YMM-350 (HUAP 75753) }\end{array}$ & $\mathrm{T}$ & Ex \\
\hline Senna hirsuta (L.) H.S. Irwin \& Barneby & YMM-273 (HUMO 36920, HUAP 75800) & $\operatorname{Ar}$ & $\mathrm{Na}$ \\
\hline Senna holwayana (Rose) H.S. Irwin \& Barneby & $\begin{array}{l}\text { GMHB-29 (HUMO 36451, HUAP 75447), RCT-3002 (HUAP } \\
\text { 75673), RCT-3058 (HUMO 36974, HUAP 75719) }\end{array}$ & $\operatorname{Ar}$ & $\mathrm{Na}$ \\
\hline Senna occidentalis (L.) Link & GMHB-370 (HUAP 75668) & $\operatorname{Ar}$ & $\mathrm{Na}$ \\
\hline Senna skinneri (Benth.) H.S. Irwin \& Barneby & GMHB-372 (HUMO 36357, HUAP 75669) & $\mathrm{Ar}$ & $\mathrm{Na}$ \\
\hline $\begin{array}{l}\text { Senna wislizeni (A. Gray) H.S. Irwin \& Barneby var. } \\
\text { pringlei (Rose) H.S. Irwin \& Barneby }\end{array}$ & $\begin{array}{l}\text { GMHB-158 (HUMO 36641, HUAP 75526), GMHB-371A } \\
\text { (HUMO 36468, HUMO 37622, HUAP 75666) }\end{array}$ & $\operatorname{Ar}$ & $\mathrm{Na}$ \\
\hline Stylosanthes humilis Kunth & GMHB-330A (HUMO 37661), YMM-290 (HUMO 36927) & $\mathrm{H}$ & $\mathrm{Na}$ \\
\hline Vachellia farnesiana (L.) Wight \& Arn. & $\begin{array}{l}\text { GMHB-325 (HUMO 36359, HUAP 75640), YMM-274 } \\
\text { (HUMO 36918, HUAP 75801) }\end{array}$ & A & $\mathrm{Na}$ \\
\hline $\begin{array}{l}\text { Vachellia pennatula (Schltdl. \& Cham.) Seigler \& } \\
\text { Ebinger }\end{array}$ & $\begin{array}{l}\text { GMHB-60A (HUMO 36455, HUAP 75468), RCT-3045 } \\
\text { (HUMO 36709, HUAP 75710) }\end{array}$ & $A$ & $\mathrm{Na}$ \\
\hline Zapoteca formosa (Kunth) H.M. Hern. & $\begin{array}{l}\text { AFM-397 (HUMO 36704, HUAP 75867), GMHB-338 } \\
\text { (HUMO 36420, HUAP 75647) }\end{array}$ & $\operatorname{Ar}$ & $\mathrm{Na}$ \\
\hline Zornia thymifolia Kunth & $\begin{array}{l}\text { GMHB-214 (HUMO 36688, HUAP 75565), GMHB-355 } \\
\text { (HUMO 36864) }\end{array}$ & $\mathrm{H}$ & $\mathrm{Na}$ \\
\hline \multicolumn{4}{|l|}{ Gentianaceae } \\
\hline \multicolumn{4}{|l|}{ Heliotropiaceae } \\
\hline Heliotropium procumbens Mill. & $\begin{array}{l}\text { AFM-354 (HUMO 36437), GMHB-80 (HUMO 36437), } \\
\text { GMHB-228 (HUMO 36696, HUAP 75573) }\end{array}$ & $\mathrm{H}$ & $\mathrm{Na}$ \\
\hline Myriopus volubilis (L.) Small & $\begin{array}{l}\text { AFM-345 (HUMO 36613, HUAP 75838), GMHB-31 (HUMO } \\
\text { 36496) }\end{array}$ & $\mathrm{T}$ & $\mathrm{Na}$ \\
\hline Tournefortia mutabilis Vent. & $\begin{array}{l}\text { AFM-324 (HUMO 36586, HUAP 75824), RCT-3087 (HUAP } \\
75738 \text { ) }\end{array}$ & $\mathrm{H}$ & $\mathrm{Na}$ \\
\hline \multicolumn{4}{|l|}{ Hernandiaceae } \\
\hline Gyrocarpus jatrophifolius Domin & $\begin{array}{l}\text { GMHB-317A (HUMO 36405, HUAP 75633), RCT-3061 } \\
\text { (HUMO 36961, HUAP 75721) }\end{array}$ & A & $\mathrm{Na}$ \\
\hline \multicolumn{4}{|l|}{ Lamiaceae } \\
\hline Salvia misella Kunth & RCT-3019A (HUMO 37642), RCT-3063 (HUMO 36949) & $\mathrm{H}$ & $\mathrm{Na}$ \\
\hline Salvia ramamoorthyana Espejo* & GMHB-261 (HUMO 36801, HUAP 75597) & $\mathrm{H}$ & $\mathrm{Na}$ \\
\hline Vitex gaumeri Greenm. & GMHB-351 (HUMO 36816, HUAP 75658) & A & $\mathrm{Na}$ \\
\hline Vitex mollis Kunth & $\begin{array}{l}\text { GMHB-137 (HUMO 37017, HUAP 75515), RCT-3052 } \\
\text { (36897, HUAP 75715) }\end{array}$ & A & $\mathrm{Na}$ \\
\hline
\end{tabular}


Apéndice: Continuación.

\begin{tabular}{|c|c|c|c|}
\hline Familias/especies & Ejemplares examinados & $\begin{array}{l}\text { Formas de } \\
\text { crecimiento }\end{array}$ & $\begin{array}{r}\text { Exótica/ } \\
\text { Nativa }\end{array}$ \\
\hline \multicolumn{4}{|l|}{ Lennoaceae } \\
\hline Lennoa madreporoides Lex. & $\begin{array}{l}\text { GMHB-305 (HUMO 36365, HUAP 75627), GMHB-348 } \\
\text { (HUMO 36867) }\end{array}$ & $P$ & $\mathrm{Na}$ \\
\hline \multicolumn{4}{|l|}{ Linderniaceae } \\
\hline $\begin{array}{l}\text { Lindernia dubia (L.) Pennell var. mexicana (S. } \\
\text { Watson) D.Q. Lewis }\end{array}$ & $\begin{array}{l}\text { GMHB-277 (HUAP 75608), RCT-3025 (HUMO 36735, } \\
\text { HUAP 75693) }\end{array}$ & $\mathrm{H}$ & $\mathrm{Na}$ \\
\hline \multicolumn{4}{|l|}{ Loasaceae } \\
\hline Gronovia scandens L. & GMHB-277 (HUMO 36768) & $\mathrm{T}$ & $\mathrm{Na}$ \\
\hline Mentzelia aspera L. & GMHB-272 (HUMO 36571, HUAP 75605) & $\mathrm{H}$ & $\mathrm{Na}$ \\
\hline Mentzelia hispida Willd. & YMM-269 (HUMO 36931, HUAP 75796) & $\mathrm{H}$ & $\mathrm{Na}$ \\
\hline \multicolumn{4}{|l|}{ Loranthaceae } \\
\hline Cladocolea dimorpha Kuijt米 & $\begin{array}{l}\text { AFM-359 (HUMO 6593, HUAP 75850), MJS-8 (HUMO } \\
\text { 37609) }\end{array}$ & P & $\mathrm{Na}$ \\
\hline Cladocolea oligantha (Standl. \& Steyerm.) Kuijt & $\begin{array}{l}\text { GMHB-262 (HUMO 36779, HUAP 75598, RCT-3051 } \\
\text { (HUMO 36909, HUAP 75714) }\end{array}$ & $\mathrm{P}$ & $\mathrm{Na}$ \\
\hline Psittacanthus palmeri (S. Watson) Barlow \& Wiens * & $\begin{array}{l}\text { GMHB-263 (HUMO 36781, HUAP 75599), RCT-3076 } \\
\text { (HUMO 36988) }\end{array}$ & $P$ & $\mathrm{Na}$ \\
\hline Struthanthus interruptus (Kunth) G. Don & GMHB-24 (HUAP 75458) & $\mathrm{P}$ & $\mathrm{Na}$ \\
\hline \multicolumn{4}{|l|}{ Lythraceae } \\
\hline Cuphea koehneana Rose* & $\begin{array}{l}\text { GMHB-231 (HUMO 36367, HUAP 75577), YMM-277 } \\
\text { (HUMO 36817, HUAP 75795) }\end{array}$ & $\mathrm{H}$ & $\mathrm{Na}$ \\
\hline Cuphea laminuligera Koehne* & $\begin{array}{l}\text { GMHB-186 (HUMO 36438), GMHB-236 (HUMO 36409, } \\
\text { HUAP 75581) }\end{array}$ & $\mathrm{H}$ & $\mathrm{Na}$ \\
\hline Heimia salicifolia Link & $\begin{array}{l}\text { AFM-351 (HUMO 36951, HUAP 75844), GMHB-79 (HUMO } \\
\text { 36368, HUAP 75476) }\end{array}$ & $\operatorname{Ar}$ & $\mathrm{Na}$ \\
\hline \multicolumn{4}{|l|}{ Malpighiaceae } \\
\hline Bunchosia canescens (Aiton) DC. * & GMHB-36 (HUMO 36502, HUAP 75446) & A & $\mathrm{Na}$ \\
\hline Galphimia paniculata Bartl. * & $\begin{array}{l}\text { RCT-3046 (HUMO 37659, HUAP 75711), RCT-3107 (HUMO } \\
\text { 36950, HUAP 75744) }\end{array}$ & $\operatorname{Ar}$ & $\mathrm{Na}$ \\
\hline Gaudichaudia diandra Nied. & $\begin{array}{l}\text { GMHB-330 (HUMO 36491, HUAP 75642), MIMV-491 } \\
\text { (HUMO 36608) }\end{array}$ & $\mathrm{T}$ & $\mathrm{Na}$ \\
\hline Heteropterys brachiata (L.) DC. & $\begin{array}{l}\text { RCT-3028 (HUMO 36574, HUAP 75697), RCT-3079 (HUMO } \\
\text { 36954) }\end{array}$ & $\mathrm{T}$ & $\mathrm{Na}$ \\
\hline Tetrapterys mexicana Hook. \& Arn. * & AFM-348 (HUMO 36599, HUAP 75841) & $\mathrm{T}$ & $\mathrm{Na}$ \\
\hline \multicolumn{4}{|l|}{ Malvaceae } \\
\hline $\begin{array}{l}\text { Allosidastrum pyramidatum (Desp. ex Cav.) Krapov., } \\
\text { Fryxell \& D.M. Bates }\end{array}$ & GMHB-7 (UAMIZ 85215), RCT-3104 (UAMIZ 85216) & $\operatorname{Ar}$ & $\mathrm{Na}$ \\
\hline Anoda crenatiflora Ortega & $\begin{array}{l}\text { GMHB-268 (HUAP 75603), GMHB-293 (HUMO 36408), } \\
\text { GMHB-294 (HUMO 36363, HUAP 75620) }\end{array}$ & $\mathrm{H}$ & $\mathrm{Na}$ \\
\hline Anoda cristata (L.) Schltdl. & RCT-3090 (HUAP 75740) & $\mathrm{H}$ & $\mathrm{Na}$ \\
\hline Byttneria aculeata (Jacq.) Jacq. & $\begin{array}{l}\text { GMHB-8 (HUAP 75465), GMHB-12 (HUAP 75462), RCT- } \\
3030 \text { (HUMO 36577, HUAP 75698) }\end{array}$ & $\operatorname{Ar}$ & $\mathrm{Na}$ \\
\hline
\end{tabular}


Apéndice: Continuación.

\begin{tabular}{|c|c|c|c|}
\hline Familias/especies & Ejemplares examinados & $\begin{array}{l}\text { Formas de } \\
\text { crecimiento }\end{array}$ & $\begin{array}{r}\text { Exótica/ } \\
\text { Nativa }\end{array}$ \\
\hline $\begin{array}{l}\text { Ceiba aesculifolia (Kunth) Britten \& Baker f. subsp. } \\
\text { parvifolia (Rose) P.E. Gibbs \& Semir }\end{array}$ & GMHB-49 (HUMO 36371, HUAP 75438) & A & $\mathrm{Na}$ \\
\hline Guazuma ulmifolia Lam. var. ulmifolia & GMHB-173 (HUMO 36651, HUAP 75534) & A & $\mathrm{Na}$ \\
\hline Herissantia crispa (L.) Brizicky & $\begin{array}{l}\text { GMHB-56 (HUMO 36373), GHMB-148B (HUMO 36374), } \\
\text { GMHB-213 (HUMO 36392), GMHB-274B (HUAP 75607), } \\
\text { GMHB-275B (HUMO 36767) }\end{array}$ & $\mathrm{H}$ & $\mathrm{Na}$ \\
\hline Hibiscus jaliscensis Fryxell & AFM-406 (HUMO 36723, HUAP 75869) & $\mathrm{H}$ & $\mathrm{Na}$ \\
\hline Melochia tomentosa $\mathrm{L}$. & $\begin{array}{l}\text { AFM-323 (HUMO 36585, HUAP 75823), AFM-402 (HUMO } \\
\text { 37656), GMHB-90 (HUMO 36400, HUAP 75484), GMHB- } \\
207 \text { (HUMO 36681, HUAP 75557), GMHB } 176 \text { (HUMO } \\
36375 \text { ) }\end{array}$ & $\mathrm{H}$ & $\mathrm{Na}$ \\
\hline Sida ciliaris L. & $\begin{array}{l}\text { MIMV-488 (HUMO 36601, HUAP 75873), GMHB-329 } \\
\text { (HUMO 36507) }\end{array}$ & $\mathrm{H}$ & $\mathrm{Na}$ \\
\hline Sida glabra Mill. & $\begin{array}{l}\text { GMHB-345A (HUMO 36466), RCT-3054 (36982, HUAP } \\
\text { 75745), RCT-3092 (HUMO 36985), RCT-3098 (HUAP } \\
\text { 75747) }\end{array}$ & $\mathrm{H}$ & $\mathrm{Na}$ \\
\hline Sida nesogena I.M. Johnst. * & RCT-3041 (HUMO 36787, HUAP 75707) & $\mathrm{H}$ & $\mathrm{Na}$ \\
\hline Sida spinosa $\mathrm{L}$. & AFM-322 (HUMO 36587) & $\mathrm{H}$ & $\mathrm{Na}$ \\
\hline Waltheria indica $\mathrm{L}$. & $\begin{array}{l}\text { GMHB-122 (HUMO 37018, HUAP 75504), GMHB-246 } \\
\text { (HUMO 36646, HUAP 75588) }\end{array}$ & $\mathrm{H}$ & $\mathrm{Na}$ \\
\hline Wissadula excelsior (Cav.) C. Presl $\bullet$ & YMM-320 (HUMO 36934, HUAP 75775) & $\mathrm{H}$ & $\mathrm{Na}$ \\
\hline Meliaceae & & & $\mathrm{Na}$ \\
\hline Trichilia americana (Sessé \& Moc.) T.D. Penn. & GMHB-15 (HUMO 36799, HUAP 75460) & A & $\mathrm{Na}$ \\
\hline Trichilia hirta L. & $\begin{array}{l}\text { GMHB-102 (HUMO 36994, HUAP 75493), RCT-3077 } \\
\text { (HUMO 36975), YMM-347 (HUMO 36814, HUAP 75793) }\end{array}$ & A & $\mathrm{Na}$ \\
\hline \multicolumn{4}{|l|}{ Menispermaceae } \\
\hline Odontocarya mexicana Barneby $* \bullet$ & $\begin{array}{l}\text { AFM-413 (HUMO 37612), AFM-414 (HUMO 37614), } \\
\text { MIMV-478 (HUMO 37649) }\end{array}$ & $\mathrm{T}$ & $\mathrm{Na}$ \\
\hline \multicolumn{4}{|l|}{ Molluginaceae } \\
\hline Mollugo verticillata $\mathrm{L}$. & AFM-355 (HUMO 36589), AFM-389 (HUMO 36875) & $\mathrm{H}$ & Ex \\
\hline \multicolumn{4}{|l|}{ Moraceae } \\
\hline Ficus cotinifolia Kunth & $\begin{array}{l}\text { AFM-375 (HUMO 36763, HUAP 75855), GMHB-370 } \\
\text { (HUMO 36483), RCT-3108 (HUMO 37652), RCT-3029 } \\
\text { (HUMO 36597, HUAP 75699) }\end{array}$ & A & $\mathrm{Na}$ \\
\hline Ficus crocata (Miq.) Miq. & $\begin{array}{l}\text { AFM-360 (HUMO 36600, HUAP 75851), AFM-382 (HUMO } \\
\text { 37610), GMHB-22 (HUMO 36797, HUAP 75459) }\end{array}$ & $A$ & $\mathrm{Na}$ \\
\hline Ficus petiolaris Kunth $*$ & GMHB-128 (HUMO 37012, HUAP 75508) & A & $\mathrm{Na}$ \\
\hline \multicolumn{4}{|l|}{ Myrtaceae } \\
\hline Psidium guajava L. & GMHB-150 (HUMO 36828, HUAP 75518) & A & $\mathrm{Na}$ \\
\hline \multicolumn{4}{|l|}{ Namaceae } \\
\hline Nama jamaicensis L. $\bullet$ & AFM-333 (HUAP 75830), RCT-3093 (HUMO 36977) & $\mathrm{H}$ & $\mathrm{Na}$ \\
\hline Nama origanifolia Kunth* & $\begin{array}{l}\text { AFM-349 (HUMO 36625, HUAP 75842), GMHB-64 (HUMO } \\
\text { 37654) }\end{array}$ & $\mathrm{H}$ & $\mathrm{Na}$ \\
\hline
\end{tabular}


Apéndice: Continuación.

\begin{tabular}{|c|c|c|c|}
\hline Familias/especies & Ejemplares examinados & $\begin{array}{l}\text { Formas de } \\
\text { crecimiento }\end{array}$ & $\begin{array}{r}\text { Exótica/ } \\
\text { Nativa }\end{array}$ \\
\hline \multicolumn{4}{|l|}{ Nyctaginaceae } \\
\hline Boerhavia coccinea Mill. & GMHB-101 (HUMO 36995, HUAP 75492) & $\mathrm{H}$ & $\mathrm{Na}$ \\
\hline Guapira petenensis (Lundell) Lundell & $\begin{array}{l}\text { GMHB-134B (HUMO 36838, HUAP 75513), MJS-3 (HUMO } \\
\text { 37631) }\end{array}$ & $A$ & $\mathrm{Na}$ \\
\hline Mirabilis longiflora L. & GMHB-114 (HUMO 37007) & $\mathrm{H}$ & $\mathrm{Na}$ \\
\hline Mirabilis viscosa Cav. & GMHB-61 (HUMO 36448) & $\mathrm{H}$ & $\mathrm{Na}$ \\
\hline \multicolumn{4}{|l|}{ Onagraceae } \\
\hline Lopezia miniata Lag. ex DC. & $\begin{array}{l}\text { MJS-268 (HUMO 37608), RCT-3039 (HUMO 36752, HUAP } \\
75705 \text { ) }\end{array}$ & $\mathrm{H}$ & $\mathrm{Na}$ \\
\hline Ludwigia octovalvis (Jacq.) P.H. Raven & $\begin{array}{l}\text { GMHB-312 (HUMO 36362, HUAP 75629), RCT-3001 } \\
\text { (HUMO 36728, HUAP 75672) }\end{array}$ & $\mathrm{H}$ & $\mathrm{Na}$ \\
\hline $\begin{array}{l}\text { Ludwigia peploides (Kunth) P.H. Raven subsp. } \\
\text { peploides }\end{array}$ & GMHB-72 (HUMO 36447, HUAP 75473) & Ac & $\mathrm{Na}$ \\
\hline Oenothera kunthiana (Spach) Munz & $\begin{array}{l}\text { AFM-334 (HUAP 75832), GMHB-125 (HUMO 36452), } \\
\text { GMHB-227 (HUMO 36453, HUAP 75572), RCT-3097 } \\
\text { (HUMO 36952) }\end{array}$ & $\mathrm{H}$ & $\mathrm{Na}$ \\
\hline \multicolumn{4}{|l|}{ Opiliaceae } \\
\hline Agonandra racemosa (DC.) Standl. & $\begin{array}{l}\text { AFM-346 (HUMO 36952, HUAP 75839), AFM-381 (HUMO } \\
\text { 36758, HUAP 75860), GHMB-14 (HUMO 37621), RCT- } \\
3075 \text { (HUAP 75732), YMM-316 (HUMO 36854, HUAP } \\
\text { 75773) }\end{array}$ & A & $\mathrm{Na}$ \\
\hline \multicolumn{4}{|l|}{ Oxalidaceae } \\
\hline Oxalis latifolia Kunth & GMHB-87 (HUMO 36446, HUAP 75481) & $\mathrm{H}$ & $\mathrm{Na}$ \\
\hline \multicolumn{4}{|l|}{ Papaveraceae } \\
\hline Argemone ochroleuca Sweet & AFM-336 (HUMO 36707, HUAP 75833) & $\mathrm{H}$ & $\mathrm{Na}$ \\
\hline \multicolumn{4}{|l|}{ Passifloraceae } \\
\hline Passiflora mexicana Juss. & YMM-344 (HUMO 36859, HUAP 75794) & $\mathrm{T}$ & $\mathrm{Na}$ \\
\hline Passiflora suberosa $\mathrm{L}$. & GMHB-174 (HUMO 36666, HUAP 75535) & $\mathrm{T}$ & $\mathrm{Na}$ \\
\hline \multicolumn{4}{|l|}{ Petiveriaceae } \\
\hline Rivina humilis L. & MIMV-480 (HUMO 36603, HUAP 75872) & $\mathrm{H}$ & $\mathrm{Na}$ \\
\hline \multicolumn{4}{|l|}{ Picramniaceae } \\
\hline Alvaradoa amorphoides Liebm. & RCT-3084 (HUMO 36965, HUAP 75736) & $\mathrm{Ar}$ & $\mathrm{Na}$ \\
\hline \multicolumn{4}{|l|}{ Plantaginaceae } \\
\hline $\begin{array}{l}\text { Mecardonia procumbens (Mill.) Small var. } \\
\text { procumbens }\end{array}$ & $\begin{array}{l}\text { AFM-331 (HUMO 36622), GMHB-171 (HUMO 36652), } \\
\text { GMHB-120B (HUMO 37009), YMM-291 (HUMO 36928) }\end{array}$ & $\mathrm{H}$ & $\mathrm{Na}$ \\
\hline Russelia coccinea (L.) Wettst. & GMHB-88 (HUMO 36457, HUAP 75482) & $\mathrm{H}$ & $\mathrm{Na}$ \\
\hline Schistophragma mexicanum Benth. ex D. Dietr. & $\begin{array}{l}\text { GMHB-319A (HUMO 36464, HUAP 75636), GMHB-357 } \\
\text { (HUMO 36863), MJS-13 (HUMO 37617), YMM-352 } \\
\text { (HUMO 36847, HUAP 75754) }\end{array}$ & $\mathrm{H}$ & $\mathrm{Na}$ \\
\hline \multicolumn{4}{|l|}{ Plumbaginaceae } \\
\hline Plumbago pulchella Boiss. * & RCT-3091 (HUMO 36973) & $\mathrm{Ar}$ & $\mathrm{Na}$ \\
\hline Plumbago zeylanica L. & AFM-376 (HUMO 36627) & $\mathrm{H}$ & $\mathrm{Na}$ \\
\hline
\end{tabular}


Apéndice: Continuación.

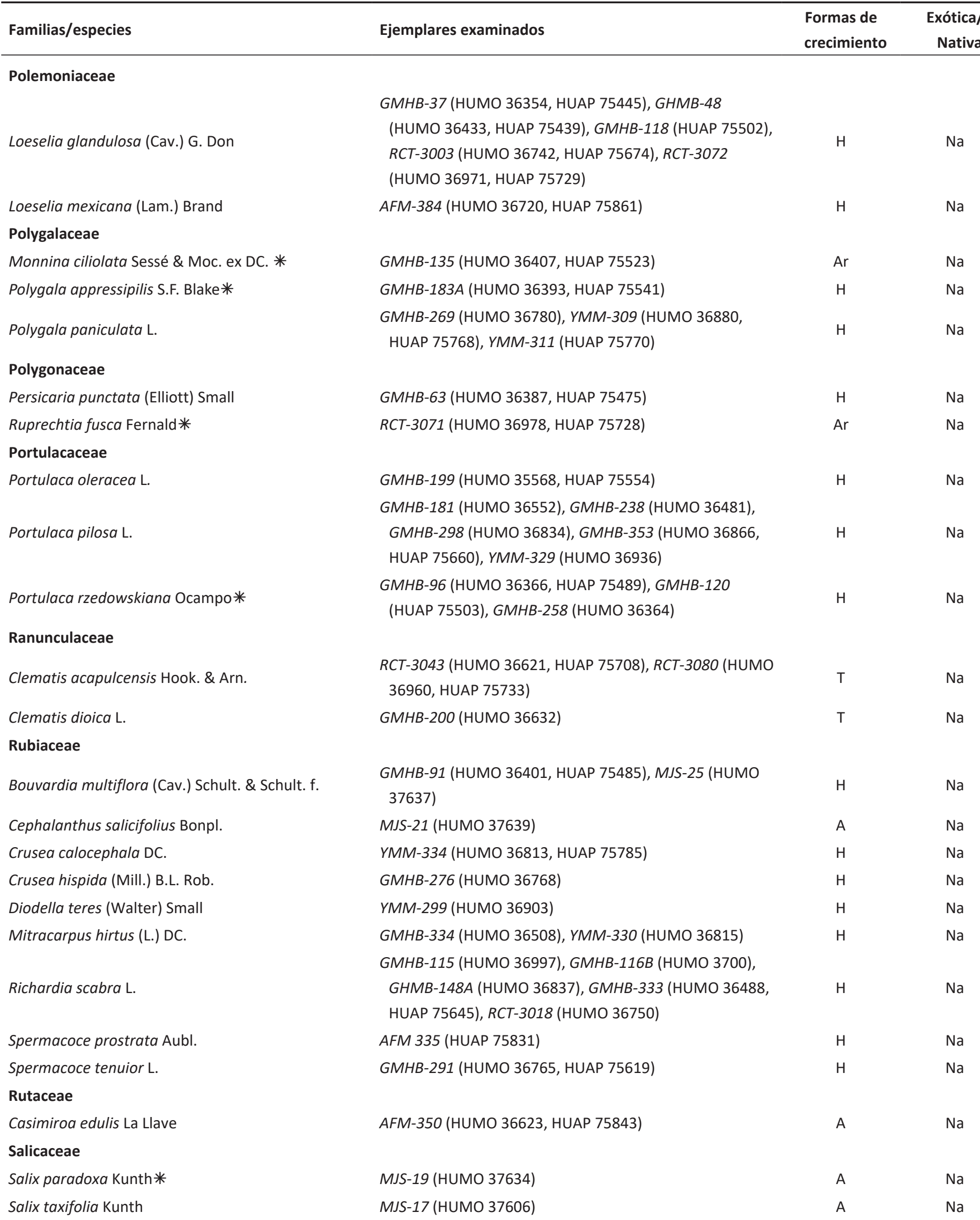


Apéndice: Continuación.

\begin{tabular}{|c|c|c|c|}
\hline Familias/especies & Ejemplares examinados & $\begin{array}{l}\text { Formas de } \\
\text { crecimiento }\end{array}$ & $\begin{array}{r}\text { Exótica/ } \\
\text { Nativa }\end{array}$ \\
\hline Xylosma flexuosa (Kunth) Hemsl. & AFM-325 (HUMO 36579, HUAP 75825) & A & $\mathrm{Na}$ \\
\hline \multicolumn{4}{|l|}{ Santalaceae } \\
\hline Phoradendron decipiens Kuijt米 & GMH-62 (HUMO 36394) & $\mathrm{P}$ & $\mathrm{Na}$ \\
\hline Phoradendron robinsonii Urb. & $\begin{array}{l}\text { GMH-133 (HUMO 37022, HUAP 75512), YMM-305 } \\
\text { (HUMO 36895) }\end{array}$ & $\mathrm{P}$ & $\mathrm{Na}$ \\
\hline \multicolumn{4}{|l|}{ Sapindaceae } \\
\hline Cardiospermum halicacabum L. & $\begin{array}{l}\text { AFM-368 (HUMO 37650), GHMB-92 (HUMO 36406), } \\
\text { GMHB-343 (HUMO 36498, HUAP 75651), RCT-3059 } \\
\text { (HUMO 36891, HUAP 75720) }\end{array}$ & $\mathrm{T}$ & $\mathrm{Na}$ \\
\hline Serjania triquetra Radlk. & GMHB-234 (HUMO 36638, HUAP 75580) & $\mathrm{T}$ & $\mathrm{Na}$ \\
\hline Serjania sp. & GMHB-17 (HUMO 36805), RCT-3016 (HUAP 75686) & $\mathrm{T}$ & $\mathrm{Na}$ \\
\hline \multicolumn{4}{|l|}{ Scrophulariaceae } \\
\hline Buddleja sessiliflora Kunth & $\begin{array}{l}\text { AFM-321 (HUMO 36590, HUAP 75822), RCT-3088 (HUAP } \\
\text { 75739) }\end{array}$ & $\mathrm{Ar}$ & $\mathrm{Na}$ \\
\hline \multicolumn{4}{|l|}{ Solanaceae } \\
\hline Cestrum dumetorum Schltdl. & AFM-343 (HUMO 36706, HUAP 75836) & $\mathrm{Ar}$ & $\mathrm{Na}$ \\
\hline Solanum adscendens Sendtn. & GMHB-170 (HUMO 36670, HUAP 75533) & $\mathrm{H}$ & $\mathrm{Na}$ \\
\hline Solanum angustifolium Mill. & RCT-3089 (HUAP 75746) & $\mathrm{H}$ & $\mathrm{Na}$ \\
\hline Solanum erianthum D. Don & AFM-344 (HUMO 36615, HUAP 75837) & $\mathrm{Ar}$ & $\mathrm{Na}$ \\
\hline Solanum oxycarpum Schiede* & $\begin{array}{l}\text { GMHB-206 (HUMO 36678, HUAP 7556), GMHB-279B } \\
\text { (HUMO 36772, HUAP 75610) }\end{array}$ & $\mathrm{H}$ & $\mathrm{Na}$ \\
\hline Solanum sp. & GMHB-188B (HUMO 36564, HUAP 75546) & $\mathrm{H}$ & $\mathrm{Na}$ \\
\hline \multicolumn{4}{|l|}{ Talinaceae } \\
\hline Talinum paniculatum (Jacq.) Gaertn. & $\begin{array}{l}\text { GMHB-95 (36369, HUAP 75488), GMHB-178 (HUMO } \\
\text { 36653) }\end{array}$ & $\mathrm{H}$ & $\mathrm{Na}$ \\
\hline \multicolumn{4}{|l|}{ Urticaceae } \\
\hline Pilea microphylla (L.) Liebm. & $\begin{array}{l}\text { GMHB-281 (HUMO 36796, HUAP 75611), YMM-275 } \\
\text { (HUMO 36930, HUAP 75802) }\end{array}$ & $\mathrm{H}$ & $\mathrm{Na}$ \\
\hline \multicolumn{4}{|l|}{ Verbenaceae } \\
\hline Bouchea prismatica (L.) Kuntze & GMHB-225 (HUMO 36634) & $\mathrm{H}$ & $\mathrm{Na}$ \\
\hline Lantana camara L. & GMHB-85 (HUMO 36442, HUAP 75479) & $\mathrm{H}$ & $\mathrm{Na}$ \\
\hline Lantana canescens Kunth & GMHB-145 (HUMO 36883, HUAP 75517) & $\mathrm{Ar}$ & $\mathrm{Na}$ \\
\hline Lantana velutina $\mathrm{M}$. Martens \& Galeotti & GMHB-129 (HUMO 37016, HUAP 75509) & $\mathrm{Ar}$ & $\mathrm{Na}$ \\
\hline Lippia umbellata Cav. & GMHB-23 (HUMO 36775) & A & $\mathrm{Na}$ \\
\hline \multicolumn{4}{|l|}{ Vitaceae } \\
\hline Cissus tiliacea Kunth & $\begin{array}{l}\text { GMHB-212A (HUMO 36876, HUAP 75562), GMHB-213 } \\
\text { (HUAP 75563) }\end{array}$ & $\mathrm{T}$ & $\mathrm{Na}$ \\
\hline Cissus verticillata (L.) Nicolson \& C.E. Jarvis & GMHB-159 (HUMO 36654) & $\mathrm{T}$ & $\mathrm{Na}$ \\
\hline
\end{tabular}

